\title{
Symplectic topology on subcritical manifolds
}

\author{
Paul Biran and Kai Cieliebak
}

\begin{abstract}
We introduce a new class of closed symplectic manifolds called subcritical. These manifolds are closed analogues of subcritical Stein manifolds. We study symplectic and Lagrangian embeddings into such manifolds and into their hyperplane sections.
\end{abstract}

Mathematics Subject Classification (2000). 53D12, 53D35, 53D40.

Keywords. Symplectic manifold, Lagrangian submanifold, Stein manifold, symplectic embedding.

\section{Introduction}

The study of the symplectic topology of Stein manifolds leads naturally to two distinct subclasses: subcritical and critical. A Stein manifold is called subcritical if it admits a plurisubharmonic function which has only critical points of index less than half the (real) dimension, and critical otherwise. Thanks to the special geometry, various problems of symplectic topology, such as Lagrangian embeddings, are more tractable on subcritical Stein manifolds than on critical ones with the tools presently available.

In this paper we introduce and study a new class of closed symplectic Kähler manifolds, which are in a sense "closed cousins" of subcritical Stein manifolds. These manifolds are, roughly speaking, closed symplectic Kähler manifolds $(M, \Omega)$ together with a complex hypersurface $\Sigma \subset M$ that represents the Poincaré dual to $k[\Omega]$ (for some $k>0$ ), and such that the complement $(M \backslash \Sigma, \Omega)$ is a subcritical Stein manifold (see Section 2 for the precise definition). We shall refer to such triples $(M, \Omega ; \Sigma)$ as subcritical polarizations (of degree $k$ ) of $(M, \Omega)$.

This notion gives rise to two interesting types of manifolds:

- Symplectic manifolds $(M, \Omega)$ which admit subcritical polarizations.

- Symplectic manifolds $\left(\Sigma,\left.\Omega\right|_{\Sigma}\right)$ that arise as hypersurfaces in a subcritical polarization $(M, \Omega ; \Sigma)$. 
As we shall see, manifolds of these types have remarkable symplectic properties, mainly concerning Lagrangian and symplectic embeddings.

The most basic example of a subcritical polarization is $\left(\mathbb{C} P^{n}, \sigma_{\mathbb{C} P^{n}} ; \Sigma \cong \mathbb{C} P^{n-1}\right)$, where $\Sigma \subset \mathbb{C} P^{n}$ is any linear hyperplane (see Section 2). Here $\mathbb{C} P^{n}$ is endowed with its standard complex and symplectic structures and the symplectic form $\sigma_{\mathbb{C} P^{n}}$ is normalized so that the area of a projective line is 1 . Also note that $\left.\sigma_{\mathbb{C} P^{n+1}}\right|_{\mathbb{C} P^{n}}=\sigma_{\mathbb{C} P^{n}}$, so besides $\left(\mathbb{C} P^{n}, \sigma_{\mathbb{C} P^{n}}\right)$ admitting a subcritical polarization it also arises as a hypersurface in a subcritical polarization, namely $\left(\mathbb{C} P^{n+1}, \sigma_{\mathbb{C}^{n+1}} ; \mathbb{C} P^{n}\right)$. More examples appear in Section 2 below.

In this paper we mainly focus on topological restrictions on Lagrangian embeddings into symplectic manifolds $\Sigma$ as above as well as some on aspects of symplectic embeddings into manifolds $(M, \Omega)$ that admit a subcritical polarization. We also develop some tools for constructing examples of subcritical polarizations.

\subsection{Lagrangian embeddings}

The most basic question one can ask regarding Lagrangian submanifolds is:

Given a symplectic manifold $(M, \Omega)$, what are the restrictions on the topology of its Lagrangian submanifolds?

Of course, one is mainly interested in restrictions beyond the ones arising from Lagrangian submanifolds being totally real. The first results in this direction were discovered by Gromov in [Gr-2] where he proved (among many other things) that $\mathbb{C}^{n}$ has no closed Lagrangian submanifolds $L$ with $H^{1}(L)=0$. The case of $\mathbb{C}^{n}$ has been extensively studied since then by many people (see [A-L-P] for a survey on the subject and [Oh-2] for a more updated list of references). Note that in comparison to general symplectic manifolds the case of $\mathbb{C}^{n}$ can be regarded as local (Darboux' Theorem). Of course, "local" should by no means be interpreted as easy. As a matter of fact the highly non-trivial tools required to attack this case reflect the complexity of the problem.

Lagrangian embeddings into other manifolds have been studied too, but mainly on two types of (Stein) manifolds: subcritical Stein manifolds (see [Vi-1, A-L-P, $\mathrm{B}-\mathrm{C}]$ ) and some cases of cotangent bundles (see the surveys [A-L-P, Vi-2]). In most of these results the presence of a global homogeneous structure such as a Liouville flow plays a crucial role. One can think of these results as "semi-local" in the sense that they provide information on Lagrangian embeddings into a neighbourhood of a given fixed Lagrangian submanifold or isotropic subcomplex.

In contrast to the above, the problem of Lagrangian embeddings into closed symplectic manifolds is of a more global nature, since one is not allowed to assume that the Lagrangian submanifold in question can be localized in a particular subdomain of the manifold. For example, a Lagrangian submanifold $L$ of $\mathbb{C} P^{n}$ cannot be always isotoped to lie in the affine part $\mathbb{C} P^{n} \backslash \mathbb{C} P^{n-1}$ due to topological reasons (e.g. $L=\mathbb{R} P^{n} \subset \mathbb{C} P^{n}, n \geq 2$ ). Moreover, even when such an isotopy does exist in 
the smooth category it is unknown whether or not it can be realized symplectically. Thus the problem of Lagrangian embeddings into $\mathbb{C} P^{n}$ cannot be localized or reduced to $\mathbb{C}^{n}$.

To the best of our knowledge, the only nontrivial restrictions on Lagrangian embeddings into closed manifolds are two results due to Seidel and to Viterbo. Viterbo proved (see [Vi-3], consult also [E-G-H]) that closed Lagrangian submanifolds of a uniruled Kähler manifold of complex dimension $>2$ cannot have any Riemannian metric of negative sectional curvature. Seidel [Se] proved the following: $A$ closed Lagrangian submanifold $L$ of $\left(\mathbb{C} P^{n}, \sigma_{\mathbb{C P} n}\right)$ must have $H^{1}(L ; \mathbb{Z} /(2 n+2) \mathbb{Z}) \neq 0$. Seidel obtained this result from his theory of graded Lagrangian submanifolds (see [Se]) which can be used as an algebraic "add-on" to the machinery of Floer homology. A delicate computation of this invariant in the case of $\mathbb{C} P^{n}$ gave rise to the above result. It is interesting to remark that in this case too, the presence of some global homogeneous structure - a Hamiltonian circle action in this case was crucial for putting the general theory to work.

From the study of subcritical manifolds we obtain new restrictions on Lagrangian embeddings into closed manifolds. In particular we shall recover Seidel's result and generalize it to a broader class of manifolds. Our approach is more geometric and completely differs from Seidel's. From our point of view the main relevant feature of the ambient manifold is that it appears as a hypersurface of a subcritical polarization. As already mentioned, $\mathbb{C} P^{n}$ is a particular case of this situation.

Below is a sample of our results on Lagrangian submanifolds of $\mathbb{C} P^{n} \times X$, for various types of manifolds $X$. These are all special cases of more general results described in Section 4.

Before we start, let us remark that below the $X$ "factor" is allowed to be a 0 dimensional manifold (namely a point), however we shall always assume implicitly that the $\mathbb{C} P^{n}$ factor is of positive dimension, namely $n \geq 1$. Henceforth we shall abbreviate the standard symplectic form $\sigma_{\mathbb{C} P^{n}}$ of $\mathbb{C} P^{n}$ to $\sigma$ whenever the dependence on the dimension $n$ is clear.

Theorem A. Let $\left(X^{2 m}, \omega_{X}\right)$ be either Stein or a closed symplectic manifold, and assume that $\pi_{2}(X)=0$. Then for $n \geq m=\operatorname{dim}_{\mathbb{C}} X,\left(\mathbb{C} P^{n} \times X^{2 m}, \sigma \oplus \omega_{X}\right)$ admits no simply connected closed Lagrangian submanifold.

For example, this results applies to $X^{2 m}$ being the torus $T^{2 m}$ (or more generally a product of closed Riemann surfaces of genus $>0$ ). See Section 4 for a sharper result and more examples.

Remark. The dimension restriction $n \geq m$ is sharp in the sense that Theorem A fails to be true for $m=n+1$. More precisely, let $\left(X^{2 n+2}, \omega_{X}\right)$ be any $2(n+1)$ dimensional symplectic manifold. Then for every sufficiently large $a>0,\left(\mathbb{C} P^{n} \times\right.$ $\left.X^{2 n+2}, \sigma \oplus a \omega_{X}\right)$ admits a Lagrangian $(2 n+1)$-sphere (see the construction in 
Section 4.3).

Theorem A can be refined as follows. Here and throughout this paper, $c_{1}^{X}$ denotes the first Chern class of the tangent bundle of a symplectic manifold $\left(X, \omega_{X}\right)$.

Theorem B. Let $\left(X^{2 m}, \omega_{X}\right)$ be a symplectic manifold for which $c_{1}^{X}=0$ in $H^{2}(X ; \mathbb{Z})$. Let $L \subset\left(\mathbb{C} P^{n} \times X^{2 m}, \sigma \oplus \omega_{X}\right)$ be a closed Lagrangian submanifold, where $n \geq m=\operatorname{dim}_{\mathbb{C}} X$.

i) If $X$ is closed with $\pi_{2}(X)=0$, then either $\pi_{1}(L)$ has an infinite cyclic subgroup, or $H_{1}(L ; \mathbb{Z})$ contains a non-trivial cyclic subgroup whose order divides $2 n+2$.

ii) If $\left(X, \omega_{X}\right)$ is an exact tame manifold (see Section 4.1), then either $H_{1}(L ; \mathbb{Z})$ has a non-trivial free subgroup, or it contains a non-trivial cyclic subgroup whose order divides $2 n+2$. Consequently, $H^{1}(L ; \mathbb{Z} /(2 n+2) \mathbb{Z}) \neq 0$.

Note that the special case $X=\mathrm{pt}$ is already non-trivial and recovers Seidel's result $[\mathrm{Se}]$ mentioned above. Other interesting examples of exact $X$ are $X=\mathbb{C}^{m}$ and $X=T^{*}(N)$ where $N$ is any closed manifold. As for closed $X$, the result applies for example to $X^{2 m}=T^{2 m}$ endowed with its standard symplectic structure, or more generally, with any symplectic structure for which $c_{1}$ vanishes in $H^{2}\left(T^{2 m} ; \mathbb{Z}\right)$.

Observe that Theorems $\mathrm{A}$ and $\mathrm{B}$ fail to be true if $X$ is allowed to have symplectic spheres. Indeed, $\mathbb{C} P^{n} \times \mathbb{C} P^{n}$ does admit closed simply connected Lagrangian submanifolds. For example, $\left\{(z, \bar{z}) \mid z \in \mathbb{C} P^{n}\right\} \subset \mathbb{C} P^{n} \times \mathbb{C} P^{n}$ is a Lagrangian copy of $\mathbb{C} P^{n}$. Nevertheless, we still have:

Theorem C. $\left(\mathbb{C} P^{n} \times \mathbb{C} P^{n}, \sigma \oplus \sigma\right)$ does not admit any closed Lagrangian submanifold $L$ with $H_{1}(L ; \mathbb{Z})=0$ and $H_{2}(L ; \mathbb{Z})=0$.

Combining Theorems A, B with a result of [B-C] we obtain the following description of Lagrangian embeddings into $\mathbb{C} P^{n} \times \mathbb{C}^{m}$. Here and in the following $\mathbb{C}^{m}$ is always endowed with its standard symplectic form $\omega_{\text {std }}=d x_{1} \wedge d y_{1}+\cdots+$ $d x_{m} \wedge d y_{m}$.

\section{Theorem D.}

1. For $n \geq m$, every closed Lagrangian submanifold $L$ of $\left(\mathbb{C} P^{n} \times \mathbb{C}^{m}, \sigma \oplus \omega_{\text {std }}\right)$ must satisfy $H^{1}(L ; \mathbb{Z} /(2 n+2) \mathbb{Z}) \neq 0$; in particular $H_{1}(L ; \mathbb{Z}) \neq 0$.

2. Every closed Lagrangian submanifold $L$ of $\left(\mathbb{C} P^{n} \times \mathbb{C}^{n+1}, \sigma \oplus \omega_{\text {std }}\right)$ with $H_{1}(L ; \mathbb{Z})=0$ must satisfy: $H^{1}(L ; \mathbb{Z} / 2 \mathbb{Z})=\cdots=H^{2 n}(L ; \mathbb{Z} / 2 \mathbb{Z})=0$.

The second case actually occurs: As noted above, $\mathbb{C} P^{n} \times \mathbb{C}^{n+1}$ does admit a Lagrangian $(2 n+1)$-sphere.

Finally, we describe a class of examples which are not products with $\mathbb{C} P^{n}$. Consider a smooth algebraic curve $C \subset \mathbb{C} P^{n}(n \geq 2)$, and denote coordinates on 
$\mathbb{C} P^{n}$ by $z=\left[z_{0}: \cdots: z_{n}\right]$ or $w=\left[w_{0}: \cdots: w_{n}\right]$. Put

$$
\Sigma=\left\{(z, w) \in \mathbb{C} P^{n} \times \mathbb{C} P^{n} \mid z \in C, \sum_{i=0}^{n} z_{i} w_{i}=0\right\} .
$$

Note that $\Sigma$ is a $\mathbb{C} P^{n-1}$-bundle over $C$. We endow $\Sigma$ with the symplectic form induced from $\mathbb{C} P^{n} \times \mathbb{C} P^{n}$, namely $\omega_{\Sigma}=\left.(\sigma \oplus \sigma)\right|_{\Sigma}$.

Theorem E. Let $C \subset \mathbb{C} P^{n}$ and $\Sigma$ be as above with $n \geq 2$ and genus $(C)>0$. Let $\left(X^{2 m}, \omega_{X}\right)$ be as in Theorem $A$ but with $0 \leq m<n-1$. Then $\left(\Sigma \times X, \omega_{\Sigma} \oplus \omega_{X}\right)$ admits no simply connected closed Lagrangian submanifold.

\subsection{Gromov radius}

Recall that the Gromov radius of a symplectic manifold $(M, \Omega)$ is defined as follows:

$$
\rho_{G}(M, \Omega)=\sup \left\{\pi r^{2} \mid B^{2 n}(r) \text { embeds symplectically into }(M, \Omega)\right\} .
$$

Here $B^{2 n}(r)$ stands for the closed ball of radius $r$, endowed with the standard symplectic structure.

Our study of subcritical manifolds gives rise to the following uniform bound on their Gromov radius in terms of the degree $k$.

Theorem F. Let $(M, \Omega, J)$ be a closed Kähler manifold that admits a subcritical polarization of degree $k$. Assume that one of the following conditions holds:

1. $\operatorname{dim}_{\mathbb{R}} M \leq 6$, or

2. $c_{1}^{M}=\lambda[\Omega]$ on $\pi_{2}(M)$ with $\lambda>2 k$.

Then $\rho_{G}(M, \Omega) \leq \frac{1}{k}$.

If, in addition, the subcritical polarization $(M, \Omega ; \Sigma)$ is such that the linear system of sections of the holomorphic line bundle $N_{\Sigma / M}=\left.\mathcal{O}_{M}(\Sigma)\right|_{\Sigma} \rightarrow \Sigma$ is base point free, then $\rho_{G}(M, \Omega)=\frac{1}{k}$.

For example, $(M, \Omega)$ can be taken to be (see Section 2$)$ :

- The blow-up of a $\mathbb{C} P^{3}$ along a line $P^{1}$ with $\Omega$ Poincaré dual do $\left[H_{1}\right]+\left[H_{2}\right]$, where $H_{1}$ is the proper transform of a plane containing $P^{1}$, and $H_{2}$ is the proper transform of a plane transverse to $P^{1}$.

- $\left(\mathbb{C} P^{n} \times T^{2 m}, \sigma \oplus l \omega\right)$ with $n>m$ and $l \geq 3$, where $\omega$ is any integral symplectic split form on $T^{2 m}$.

In both these examples $k=1$, and we actually have equality $\rho_{G}=1$. More examples and sharper results appear in Sections 2 and 8. 


\section{Remarks.}

1. Conditions 1-2 of Theorem $\mathrm{F}$ arise from technical reasons in the proof. We strongly believe that they can be dropped.

2. The computation of the Gromov radius in both of the above examples can be accomplished also by different (though ad hoc) techniques than the ones presented in this paper. Thus the main novelty in Theorem $\mathrm{F}$ is not so much in computing new examples, but rather in the fact that subcritical manifolds share a uniform bound on their Gromov radius.

Given a Kähler manifold $(M, \Omega, J)$ with $\Omega \in H^{2}(M ; \mathbb{Z})$ we denote by $k_{\text {hol }}(M, J,[\Omega])$ the minimal integer $k$ for which there exists a smooth and reduced complex hypersurface $\Sigma \subset M$ that represents the homology class Poincaré dual to $k[\Omega]$.

Combining Theorem $\mathrm{F}$ with results from algebraic geometry due to Ein, Küchle and Lazarsfeld [E-K-L] we get the following theorem:

Theorem G. Let $(M, \Omega, J)$ be a closed Kähler manifold that admits a subcritical polarization of degree $k$. If $(M, \Omega)$ and $k$ satisfy one of the conditions 1-2 of Theorem $F$ then $k \leq \operatorname{dim}_{\mathbb{C}} M$. In particular $k_{\mathrm{hol}}(M, J,[\Omega]) \leq \operatorname{dim}_{\mathbb{C}} M$.

\subsection{The role of subcriticality}

Before we delve into the details let us briefly outline how subcriticality is used to obtain our results.

An important feature of (complete) subcritical Stein manifolds, observed in [B-C], is that any closed Lagrangian submanifold of such a manifold can be displaced away from itself via a Hamiltonian isotopy. In particular, the phenomenon of Lagrangian intersections never occurs in such manifolds. Consequently, Lagrangian submanifolds $L \subset\left(V, \omega_{V}\right)$ of subcritical Stein manifolds enjoy the following two remarkable properties:

1. Whenever well defined, the Floer cohomology $H F^{*}(L, L)$ vanishes.

2. There exists a holomorphic disc $D \subset V$ with boundary on $L$ with "low" Maslov number $\mu(D) \leq \operatorname{dim}_{\mathbb{C}} V+1$. This is called "Maslov class rigidity" (see $[\mathrm{B}-\mathrm{C}]$ for more details).

Under suitable a priori assumptions on the Maslov index of a given Lagrangian, properties 1 and 2 impose strong restrictions on the topology of $L$ (see [B-C]).

Our study of Lagrangian submanifolds in the closed(!) subcritical context is based on the above ideas. Let $\left(\Sigma, \omega_{\Sigma}\right)$ be a closed symplectic manifold that appears as a hypersurface of a subcritical polarization, say $(M, \Omega ; \Sigma)$. In order to reach the favorite situation of a subcritical Stein manifold, we "lift" $L$ to a certain Lagrangian submanifold $\hat{L} \subset(M \backslash \Sigma, \Omega)$ which is a circle bundle over $L$. The point of this lifting construction is that now we have constructed a Lagrangian submanifold, closely related to $L$, which lies inside a subcritical Stein manifold, 
namely in $(M \backslash \Sigma, \Omega)$. Under certain assumptions on the first Chern class of $\left(\Sigma, \omega_{\Sigma}\right)$ we get strong restrictions on the topology of $\hat{L}$. As $\hat{L}$ is nothing but a circle bundle over $L$ this yields restrictions on the topology of $L$. More details on this strategy and on the "lifting" procedure are given in Section 5.2.

Subcriticality is also used in an essential way in our study of the Gromov radius. Suppose that $(M, \Omega ; \Sigma)$ is a subcritical polarization. Then by the results of [Bi-1] (see Section 3 for a summary) it follows that $(M, \Omega)$ can be decomposed into two disjoint subsets: $(M, \Omega)=\left(E_{\Sigma}, \omega_{0}\right) \amalg \Delta$, where $E_{\Sigma}$ is a disc bundle over $\Sigma$ endowed with a standard symplectic form $\omega_{0}$, and $\Delta \subset M$ is a CW-complex with $\Omega$-isotropic cells and such that $\operatorname{dim} \Delta<\frac{1}{2} \operatorname{dim} M$. In other words, none of the cells of $\Delta$ is Lagrangian.

Now given a symplectically embedded ball $B \subset M$, Gromov's h-principle implies that it possible to disjoin $B$ from $\Delta$ by a symplectic isotopy. Thus we may assume that $B \subset(M \backslash \Delta, \Omega)=\left(E, \omega_{0}\right)$. This implies that $\rho_{G}(M, \Omega)=\rho_{G}\left(E, \omega_{0}\right)$. The point of all this "acrobatics" is that the Gromov radius of $\left(E, \omega_{0}\right)$ can be estimated from above (and sometimes even exactly computed) using the theory of pseudo-holomorphic curves. This is possible due to the standard symplectic structure $\omega_{0}$ of $E$. See Section 6 and [Bi-1] for more details.

We point out that subcriticality is crucial for this type of argument to work, for otherwise the CW-complex $\Delta$ would contain also Lagrangian cells, a case in which the h-principle fails.

\subsection{Organization of the paper}

The rest of the paper is organized as follows.

In Section 2 we introduce the notion of subcritical polarizations and construct examples. The constructions are based on a "desingularization theorem", which is proved in Section 7. In Section 3 we summarize the results on decompositions of polarized manifolds that we need later in the paper.

Section 4 is concerned with Lagrangian embeddings. We state here the most general versions of our theorems in this direction, derive corollaries and list concrete examples. Theorems A, B, and $\mathrm{E}$ of the introduction are immediate consequences of the corollaries. The main theorems on Lagrangian embeddings, Theorems 4.2.1, 4.2.2, 4.2.3 as well as Theorem $\mathrm{C}$ from the introduction, are proved in Section 5 .

In Section 6 we prove the uniform bounds on the Gromov radius of subcritical manifolds formulated in Theorems F and G. Finally, in Section 8 we briefly discuss some other issues related to subcritical manifolds, such as symplectic packings and capacities.

Acknowledgements. We thank the referee for pointing out to us the reference to Laudenbach's paper [Lau]. 


\section{Subcritical polarizations}

\subsection{Setup}

Subcritical Stein manifolds. A Stein manifold is a triple $(V, J, \varphi)$ where $(V, J)$ is an open complex manifold and $\varphi: V \rightarrow \mathbb{R}$ is a smooth exhausting plurisubharmonic function. The term "exhausting" means that $\varphi$ is proper and bounded from below. "Plurisubharmonic" means that the 2 -form $\omega_{\varphi}=-d d^{\mathbb{C}} \varphi$ is a $J$-positive symplectic form, i.e. $-d d^{\mathbb{C}} \varphi(v, J v)>0$ for every $0 \neq v \in T(V)$. Unless explicitly stated, we do not assume that $(V, J, \varphi)$ is complete in the Eliashberg-Gromov [E-G] sense. We refer the reader to [El-1, El-2] for the foundations of the symplectic theory of Stein manifolds.

It is well known that any plurisubharmonic Morse function $\varphi: V \rightarrow \mathbb{R}$ must satisfy $\operatorname{index}_{p}(\varphi) \leq \frac{1}{2} \operatorname{dim}_{\mathbb{R}} V$ for all critical points $p$. We call a Stein manifold subcritical if these inequalities are strict, i.e. $\varphi$ is a Morse function all of whose critical points have $\operatorname{index}_{p}(\varphi)<\frac{1}{2} \operatorname{dim}_{\mathbb{R}} V$.

Polarized Kähler manifolds. Throughout this paper, by a Kähler manifold we mean a triple $(M, \Omega, J)$ where $(M, \Omega)$ is a closed symplectic manifold and $J$ is an (integrable) complex structure compatible with $\Omega$.

A polarized Kähler manifold ${ }^{1} \mathcal{P}=\left(M^{2 n}, \Omega, J ; \Sigma\right)$ is a Kähler manifold $(M, \Omega, J)$ with $[\Omega] \in H^{2}(M ; \mathbb{Z})$ together with a smooth and reduced complex hypersurface $\Sigma \subset M$ whose homology class $[\Sigma] \in H_{2 n-2}(M)$ represents the Poincaré dual to $k[\Omega] \in H^{2}(M)$ for some $k \in \mathbb{N}$. The number $k$ will be called the degree of the polarization $\mathcal{P}$ and denoted by $k_{\mathcal{P}}$.

The function associated with a polarization. We shall now define a distinguished plurisubharmonic function $\varphi_{\mathcal{P}}: M \backslash \Sigma \rightarrow \mathbb{R}$ which is canonically associated with the polarization $\mathcal{P}$. For this purpose let $\mathcal{L}=\mathcal{O}_{M}(\Sigma)$ be the holomorphic line bundle defined by the divisor $\Sigma$. Denote by $s: M \rightarrow \mathcal{L}$ the (unique up to a constant factor) holomorphic section whose zero set $\{s=0\}$ is $\Sigma$. Choose a hermitian metric $\|\cdot\|$ on $\mathcal{L}$, and a compatible metric connection $\nabla$ with curvature

$$
R^{\nabla}=2 \pi i k_{\mathcal{P}} \Omega \text {. }
$$

Finally, define $\varphi_{\mathcal{P}}: M \backslash \Sigma \rightarrow \mathbb{R}$ to be

$$
\varphi_{\mathcal{P}}(x)=-\frac{1}{4 \pi k_{\mathcal{P}}} \log \|s(x)\|^{2} .
$$

Note that the function $\varphi_{\mathcal{P}}:(V=M \backslash \Sigma, J) \rightarrow \mathbb{R}$ is plurisubharmonic. Indeed, a simple computation shows that $-d d^{\mathbb{C}} \varphi_{\mathcal{P}}=\Omega$. Moreover, it is not hard to see that $\varphi_{\mathcal{P}}$ is exhausting and that it has no critical points outside some compact subset of $V$ (see $[\mathrm{Bi}-1])$.

\footnotetext{
1 Note that our notion of polarized Kähler manifolds is slightly different from the one common in algebraic geometry.
} 
It is important to remark that the function $\varphi_{\mathcal{P}}$ is canonically determined by the polarization $\mathcal{P}$ up to an additive constant and does not depend on any of the choices made for $\|\cdot\|, s$ or $\nabla$. This is due to the requirement on the curvature $R^{\nabla}$ and the fact that $J$ is integrable (see [Bi-1] for more details).

Finally, let us mention that we do not assume $\varphi_{\mathcal{P}}$ to be Morse in general, although this will be the case in many of the examples below.

Subcritical polarizations. A polarization $\mathcal{P}=(M, \Omega, J ; \Sigma)$ is called subcritical if there exists a plurisubharmonic function $\varphi:(V=M \backslash \Sigma, J) \rightarrow \mathbb{R}$ such that:

1. $(V, J, \varphi)$ is a subcritical Stein manifold, namely $\varphi$ is Morse and for every $p \in \operatorname{Crit}(\varphi), \operatorname{index}_{p}(\varphi)<\frac{1}{2} \operatorname{dim}_{\mathbb{R}} V$.

2. $\varphi$ coincides with $\varphi_{\mathcal{P}}$ outside a compact subset of $V$ that contains $\operatorname{Crit}(\varphi)$. Note that this implies that $\operatorname{Crit}(\varphi)$ is finite.

In practice, it is not easy to apply this definition directly even if $\varphi=\varphi_{\mathcal{P}}$ itself satisfies the above conditions. However we shall develop below (see Section 2.3) some useful criteria for checking subcriticality.

\subsection{Examples of subcritical polarizations}

Below is a list of examples of subcritical polarizations. The construction of these examples is based on a desingularization procedure presented in Section 2.3 below. Most of these examples are a special case of more general family of subcritical polarizations which is described in Section 2.4.

2.2.1. Subcritical polarizations of $\mathbb{C} P^{n}$. Consider the complex projective space $\mathbb{C} P^{n}$ endowed with the standard complex structure $J_{\mathbb{C} P^{n}}$ and its standard symplectic Kähler form $\sigma_{\mathbb{C} P^{n}}$ normalized so that the area of each projective line is 1 . In what follows we denote by $\left[z_{0}: \ldots: z_{n}\right]$ homogeneous coordinates on $\mathbb{C} P^{n}$. On each affine chart $U_{j}=\left\{z_{j} \neq 0\right\}$ with coordinates $u_{k}=\frac{z_{k}}{z_{j}}(k \neq j)$ the form $\sigma$ is given by

$$
\sigma=\frac{i}{2 \pi} \partial \bar{\partial} \log \left(1+\sum_{k \neq j}^{n}\left|u_{k}\right|^{2}\right) .
$$

Let $\Sigma \subset \mathbb{C} P^{n}$ be any linear hyperplane (namely, defined by a linear equation). Then the polarization $\mathcal{P}=\left(\mathbb{C} P^{n}, \sigma, J_{\mathbb{C} P^{n}} ; \Sigma\right)$ is subcritical of degree $k_{\mathcal{P}}=1$.

Indeed, suppose that $\Sigma=\left\{\lambda_{0} z_{0}+\cdots+\lambda_{n} z_{n}=0\right\}$, then it is easy to see that

$$
\varphi_{\mathcal{P}}\left(\left[z_{0}: \ldots: z_{n}\right]\right)=-\frac{1}{4 \pi} \log \left(\frac{\left|\sum_{i=0}^{n} \lambda_{i} z_{i}\right|^{2}}{\sum_{i=0}^{n}\left|z_{i}\right|^{2}}\right) .
$$

A simple computation shows that this function is Morse with only one critical point, $p=\left[\bar{\lambda}_{0}: \ldots: \bar{\lambda}_{n}\right]$, whose index is 0 .

Finally, observe that $\left(\Sigma,\left.\sigma_{\mathbb{C} P^{n}}\right|_{\Sigma}\right) \cong\left(\mathbb{C} P^{n-1}, \sigma_{\mathbb{C} P^{n-1}}\right)$. As this holds for every $n \geq 1$, we conclude that $\left(\mathbb{C} P^{n}, \sigma\right)$ can also be realized as a hypersurface of a subcritical polarization. 
2.2.2. Products with $\mathbb{C} P^{n}$. Let $\left(Y^{2 m}, J_{Y}\right)$ be a closed complex manifold of complex dimension $m$, and $\mathcal{L} \rightarrow Y$ a very ample line bundle. Let $\Omega_{\mathcal{L}}$ be a $J_{Y}$-Kähler representative of $c_{1}^{\mathcal{L}}$. If $n>m$ then $\left(\mathbb{C} P^{n} \times Y^{2 m}, \sigma \oplus \Omega_{\mathcal{L}}, J_{\mathbb{C} P^{n}} \oplus J_{Y}\right)$ admits a subcritical polarization of degree 1 . The proof of this fact is given in Section 2.5 below.

A special case of this is when $Y^{2 m} \subset \mathbb{C} P^{n}$ is a closed algebraic submanifold of complex dimension $m<n$. Take $\Sigma \subset \mathbb{C} P^{n} \times Y$ to be the hypersurface

$$
\Sigma=\left\{(z, w) \in \mathbb{C} P^{n} \times \mathbb{C} P^{n} \mid w \in Y, \sum_{i=0}^{n} z_{i} w_{i}=0\right\} .
$$

Then $\left(\mathbb{C} P^{n} \times Y^{2 m},\left.\sigma \oplus \sigma\right|_{Y}, J_{\mathbb{C} P^{n}} \oplus J_{Y} ; \Sigma\right)$ is a subcritical polarization.

2.2.3. Subcritical polarizations of $\mathbb{C} P^{n} \times \mathbb{C} P^{m}$. In view of the preceding example, for $n>m,\left(\mathbb{C} P^{n} \times \mathbb{C} P^{m}, \sigma \oplus \sigma\right)$ admits a subcritical polarization with the hypersurface

$$
\Sigma=\left\{(z, w) \in \mathbb{C} P^{n} \times \mathbb{C} P^{m} \mid \sum_{i=0}^{m} z_{i} w_{i}=0\right\},
$$

where $\left[z_{0}: \cdots: z_{n}\right],\left[w_{0}: \cdots: w_{m}\right]$ are homogeneous coordinates on each factor of $\mathbb{C} P^{n} \times \mathbb{C} P^{m}$. On the other hand, it is possible to prove that $\mathbb{C} P^{n} \times \mathbb{C} P^{n}$ has no subcritical polarizations at all (see $[\mathrm{Bi}-1])$.

2.2.4. Blow-ups. Let $k \geq 1, m \geq 0$ be integers. Fix a (complex) $(k-1)$-dimensional linear subspace $P_{0}^{k-1} \subset \mathbb{C} P^{m+k}$ and let

$$
X_{k, m}=\mathrm{Bl}_{P_{0}^{k-1}} \mathbb{C} P^{m+k}
$$

be the blow-up of $\mathbb{C} P^{m+k}$ along $P_{0}^{k-1}$. We denote by $J_{k, m}$ the obvious complex structure on $X_{k, m}$. Consider now the following two divisors in $X_{k, m}$ :

- $H_{1}$ - the proper transform of a linear hyperplane intersecting $P_{0}^{k-1}$ transversally.

- $\mathrm{H}_{2}$ - the proper transform of a linear hyperplane in $\mathbb{C} P^{m+k}$ that contains $P_{0}^{k-1}$

Suppose now that $k>m$. Then there exists a $J_{k, m}$-Kähler form $\Omega_{k, m}$ on $X_{k, m}$ and a smooth and reduced hypersurface $\Sigma \in\left|H_{1}+H_{2}\right|$ such that:

1. $\left[\Omega_{k, m}\right]$ is Poincaré dual to $\left[H_{1}\right]+\left[H_{2}\right]$.

2. The degree 1 polarization $\mathcal{P}=\left(X_{k, m}, \Omega_{k, m}, J_{k, m} ; \Sigma\right)$ is subcritical.

The proof is given in Section 2.5 below.

\subsection{Desingularization}

Our main tool in constructing subcritical polarizations is a desingularization procedure which we now describe. 
Let $(M, J)$ be a closed complex manifold and $\mathcal{L}_{1}, \mathcal{L}_{2} \rightarrow M$ holomorphic line bundles such that $\mathcal{L}=\mathcal{L}_{1} \otimes \mathcal{L}_{2}$ is ample. Let $\|\cdot\|, \nabla$ be a hermitian metric and a compatible connection on $\mathcal{L}$ such that the corresponding curvature form $\Omega_{\mathcal{L}}=\frac{1}{2 \pi i} R^{\nabla}$ is positive. Suppose we are given:

- Holomorphic sections $s_{i}: M \rightarrow \mathcal{L}_{i}(i=1,2)$ transverse to the zero sections and such that the codimension-1 complex hypersurfaces $\Sigma_{i}=\left\{s_{i}=0\right\}$ intersect transversally in the codimension-2 complex submanifold $D=\Sigma_{1} \cap \Sigma_{2}$.

- A holomorphic section $s_{0}: M \rightarrow \mathcal{L}$ with $\left.s_{0}\right|_{D}: D \rightarrow \mathcal{L}$ being transverse to the zero section so that $Z=D \cap\left\{s_{0}=0\right\}$ is a codimension-3 complex submanifold.

Note that $\left(M \backslash\left(\Sigma_{1} \cup \Sigma_{2}\right), J\right)$ and $\left(D \backslash Z,\left.J\right|_{D}\right)$ are Stein manifolds since $\Sigma_{1}+\Sigma_{2} \subset M$ and $Z \subset D$ are both ample divisors.

Theorem 2.3.1 (Desingularization Theorem). Suppose that the following conditions are satisfied:

1. $\left(M \backslash\left(\Sigma_{1} \cup \Sigma_{2}\right), J,-\log \left\|s_{1} \otimes s_{2}\right\|^{2}\right)$ is a subcritical Stein manifold.

2. $\left(D \backslash Z,\left.J\right|_{D},-\log \left\|s_{0}\right\|^{2}\right)$ is a subcritical Stein manifold.

Then for $\epsilon>0$ sufficiently small the following holds:

1. $s=\left(s_{1} \otimes s_{2}+\epsilon s_{0}\right): M \rightarrow \mathcal{L}$ is transverse to the zero section so that $\Sigma=\{s=0\}$ is a smooth and reduced hypersurface.

2. The polarization $\mathcal{P}=\left(M, \Omega_{\mathcal{L}}, J ; \Sigma\right)$ is subcritical.

Remark. Note that by definition a zero-dimensional manifold (namely a point or a bunch of points) is not subcritical. Therefore the conditions of the theorem can be satisfied only if $\operatorname{dim}_{\mathbb{C}} M \geq 3$.

The proof of Theorem 2.3.1 is postponed until Section 7 below. We now turn to some applications of this theorem.

\subsection{Projective bundles}

Let us first fix a few algebro-geometric notations. Let $\left(Y, J_{Y}\right)$ be a closed complex manifold and $E \rightarrow Y$ a holomorphic vector bundle. We denote by $\mathbb{P}(E) \stackrel{\pi}{\rightarrow} Y$ the corresponding projective bundle. We remark that here we adopt Fulton's convention $[\mathrm{Fu}]$ rather than the "French" one, namely the fibre over $y \in Y$ is the complex projectivization of the fibre $E_{y}$ (that is the space of complex lines) rather than the projectivization of the dual of $E_{y}$.

The complex manifold $\mathbb{P}(E)$ comes naturally equipped with a holomorphic line bundle $\mathcal{O}_{E}(1) \rightarrow \mathbb{P}(E)$ (called the hyperplane bundle) whose fibre over the line $l_{y} \in \mathbb{P}\left(E_{y}\right)$ is just the dual $l_{y}^{*}=\operatorname{Hom}_{\mathbb{C}}\left(l_{y}, \mathbb{C}\right)$.

We are now in the position to describe a new family of subcritical polarizations. Let $\left(Y^{2 m}, J_{Y}\right)$ be a closed complex manifold of complex dimension $m$ and $E \rightarrow Y$ 
a rank-l holomorphic vector bundle. Consider the projective bundle

$$
X_{E, k}=\mathbb{P}\left(E \oplus \mathbb{C}^{k}\right) \stackrel{\pi}{\rightarrow} Y,
$$

where $\mathbb{C}^{k}$ stands for the trivial rank- $k$ holomorphic vector bundle over $Y$. We denote by $J_{X_{E, k}}$ the induced complex structure on $X_{E, k}$.

Suppose in addition that:

1. $E \rightarrow Y$ is semi-negative in the sense of Griffiths (see [Gri, Dem]).

2. We are given an ample line bundle $\mathcal{L} \rightarrow Y$.

Under these assumptions we shall now endow $X_{E, k}$ with a Kähler form $\Omega_{E, k, \mathcal{L}}$ which represents the first Chern class of the line bundle $\mathcal{O}_{E \oplus \mathbb{C}^{k}}(1) \otimes \pi^{*} \mathcal{L}$.

For brevity we write $E_{k}=E \oplus \mathbb{C}^{k}$. Choose a hermitian metric $\|\cdot\|_{E}$ on $E$ which has semi-negative curvature. The hermitian metric $\|\cdot\|_{E}$ induces a hermitian metric on $\mathcal{O}_{E_{k}}(1)$. We claim that the curvature of $\mathcal{O}_{E_{k}}(1)$ is semi-positive. Indeed, there exists a natural surjection $\pi^{*}\left(E_{k}^{*}\right) \rightarrow \mathcal{O}_{E_{k}}(1)$ hence $\mathcal{O}_{E_{k}}(1)$ is a quotient of the pull back bundle $\pi^{*}\left(E_{k}^{*}\right)$. From our assumptions it follows that $\pi^{*}\left(E_{k}^{*}\right)$ has semi-positive curvature, therefore the same holds also for $\mathcal{O}_{E_{k}}(1)$ (see $[\mathrm{G}-\mathrm{H}]$ ). Denote by $\eta_{E, k}=\frac{1}{2 \pi i} R^{\mathcal{O}_{E_{k}}(1)}$ the corresponding semi-positive curvature form.

Next, endow $\mathcal{L}$ with a hermitian metric and connection $\|\cdot\|_{\mathcal{L}}, \nabla$ so that $\Omega_{\mathcal{L}}=$ $\frac{1}{2 \pi i} R^{\nabla}$ is a Kähler form on $Y$. We claim that form

$$
\Omega_{E, k, \mathcal{L}}=\pi^{*} \Omega_{\mathcal{L}}+\eta_{E, k}
$$

is Kähler. Indeed, $\eta_{E, k}$ restricts to a Kähler form on each of the fibres of $\mathbb{P}\left(E_{k}\right) \rightarrow$ $Y$ (this form corresponds to the standard Kähler form on $\mathbb{C} P^{l+k-1}$ under an identification of a fibre $E_{y} \oplus \mathbb{C}^{k}$ with $\mathbb{C}^{l+k}$ equipped with its standard hermitian metric). Since $\Omega_{\mathcal{L}}$ is Kähler and both of the forms $\pi^{*} \Omega_{\mathcal{L}}$ and $\eta_{E, k}$ are semi-positive it easily follows that their sum is strictly positive. This concludes the construction of the symplectic Kähler form $\Omega_{E, k, \mathcal{L}}$.

Before we proceed, observe that the line bundle $\mathcal{O}_{E_{k}}(1)$ has $k$ natural sections induced from projections of $E_{k}=E \oplus \mathbb{C}^{k}$ onto the components of $\mathbb{C}^{k}$. We denote these sections by $\sigma_{0}, \ldots, \sigma_{k-1}$.

Jointly transverse sections. The following definition is analogous to the notion of complete intersections. Let $\left(Y, J_{Y}\right)$ be a closed complex manifold endowed with an ample line bundle $\mathcal{L} \rightarrow\left(Y, J_{Y}\right)$. Suppose we are given $m+1$ holomorphic sections $s_{0}, \ldots, s_{m}: Y \rightarrow \mathcal{L}$. We say that they are jointly transverse if $s_{0}$ is transverse to the zero section of $\mathcal{L}$ and for every $1 \leq i \leq m$, the section $s_{i}$ is transverse to the complex submanifold $D_{i}=\left\{s_{0}=\cdots=s_{i-1}=0\right\}$. Note that in this case $D_{i}$ is a codimension- $i$ complex submanifold of $\left(Y, J_{Y}\right)$.

An important example is when $\mathcal{L}$ is a very ample line bundle. In this case there always exist $\operatorname{dim}_{\mathbb{C}} Y+1$ jointly transverse sections.

Theorem 2.4.1. Let $E \rightarrow Y^{2 m}, \mathcal{L} \rightarrow Y^{2 m}$, and $\left(X_{E, k}, \Omega_{E, k, \mathcal{L}}, J_{X_{E, k}}\right)$ be as above and assume that:

- either $k>m+1$, 
- or $k=m+1$ and $l \geq 1$.

Suppose also that $\mathcal{L} \rightarrow Y$ admits $m+1$ jointly transverse sections $s_{0}, \ldots, s_{m}$ : $Y \rightarrow \mathcal{L}$. Then there exist constants $\epsilon_{i}>0$ such that:

1. $s=\left(\sigma_{0} \otimes \pi^{*} s_{0}+\sum_{i=1}^{m} \epsilon_{i} \sigma_{i} \otimes \pi^{*} s_{i}\right): X_{E, k} \rightarrow \mathcal{O}_{E_{k}}(1) \otimes \pi^{*} \mathcal{L}$ is transverse to the zero section so that $\Sigma=\{s=0\}$ is a smooth and reduced hypersurface.

2. The polarization $\mathcal{P}=\left(X_{E, k}, \Omega_{E, k, \mathcal{L}}, J_{X_{E, k}} ; \Sigma\right)$ is subcritical.

Proof. The proof proceeds by induction over $m=\operatorname{dim}_{\mathbb{C}} Y$.

For $m=0$, we may assume that $Y$ is a single point. Then $E \cong \mathbb{C}^{l}$, $X_{E, k} \cong \mathbb{C} P^{k+l-1}, 0 \neq s_{0} \in \mathcal{L} \cong \mathbb{C}$, and $\sigma_{0} \otimes \pi^{*} s_{0}$ is a nontrivial section of the line bundle $\mathcal{O}(1) \otimes \mathbb{C} \cong \mathcal{O}(1) \rightarrow \mathbb{C} P^{k+l-1}$. Thus $\left(X_{E, k}, \Omega_{E, k, \mathcal{L}}, J_{X_{E, k}} ; \Sigma\right)=$ $\left(\mathbb{C} P^{k+l-1}, \sigma, J_{\mathbb{C} P^{k+l-1}} ; \Sigma\right)$ is just the subcritical polarization of $\mathbb{C} P^{k+l-1}$ discussed above.

Assume now that $m \geq 1$ and the theorem holds for $m-1$. The sections $\sigma_{0}: X_{E, k} \rightarrow \mathcal{O}_{E_{k}}(1)$ and $\pi^{*} s_{0}: X_{E, k} \rightarrow \pi^{*} \mathcal{L}$ are transverse to the zero section. Moreover, their zero sets intersect transversally in the codimension-2 submanifold

$$
X_{\tilde{E}, k-1}=\mathbb{P}\left(\tilde{E} \oplus \mathbb{C}^{k-1}\right) \rightarrow \tilde{Y},
$$

where $\tilde{Y}=\left\{s_{0}=0\right\} \subset Y$ and $\tilde{E}=\left.E\right|_{\tilde{Y}}$. Now $X_{\tilde{E}, k-1} \rightarrow Y$ with the restrictions of $\sigma_{1}, \ldots, \sigma_{k}$ and $s_{1}, \ldots, s_{k}$ satisfies the assumptions of the theorem for $(m-1, k-1, l)$. By the induction hypothesis there exist positive constants $\tilde{\epsilon}_{2}, \ldots, \tilde{\epsilon}_{m}$ such that

$$
\tilde{s}=\sigma_{1} \otimes \pi^{*} s_{1}+\sum_{i=2}^{m} \tilde{\epsilon}_{i} \sigma_{i} \otimes \pi^{*} s_{i}
$$

is transverse to $X_{\tilde{E}, k-1}$ and induces a subcritical polarization on $X_{\tilde{E}, k-1}$. So the theorem will follow from Theorem 2.3.1 once we have shown that

$$
\left(X_{E, k} \backslash\left(\left\{\sigma_{0}=0\right\} \cup\left\{\pi^{*} s_{0}=0\right\}\right), J_{X_{E, k}}, \phi=-\log \left\|\sigma_{0} \otimes \pi^{*} s_{0}\right\|^{2}\right)
$$

is a subcritical Stein manifold.

To see this, denote by $y, e, z=\left(z_{1}, \ldots, z_{k}\right)$ coordinates on $Y, E, \mathbb{C}^{k}$ respectively. Then $\phi(y,[e: z])=\phi_{1}(y,[e: z])+\phi_{2}(y)$ where

$$
\phi_{1}(y,[e: z])=-\log \left(\frac{\left|z_{1}\right|^{2}}{\|z\|^{2}+\|e\|^{2}}\right) \quad \text { and } \quad \phi_{2}(y)=-\log \left\|\sigma_{0}(y)\right\|^{2} .
$$

The restriction of $\phi$ to a fibre $\pi^{-1}(y)$ has a unique critical point $[e: z]=[0:(1:$ $0: \cdots: 0)]$ which corresponds to the minimum of $\phi_{1}$. So the critical points of $\phi$ are precisely the points $(y,[0:(1: 0: \cdots: 0)])$ with $d \phi_{2}(y)=0$. After perturbing the metric on $\mathcal{L}$ we may assume that the critical points of $\phi_{2}$ are non-degenerate. Then the critical points of $\phi$ are non-degenerate of index

$$
\operatorname{ind}_{(y,[0:(1: 0: \cdots: 0)])} \phi=\operatorname{ind}_{y} \phi_{2}+0 \leq m<\operatorname{dim}_{\mathbb{C}} X_{E, k},
$$

hence they are subcritical. 


\subsection{Proofs of the statements in examples 2.2.2 and 2.2.4}

The example given in 2.2.2 is a special case of Theorem 2.4.1. In fact, Theorem 2.4.1 implies the following slightly stronger statement:

Corollary 2.5.1. Let $\left(Y^{2 m}, J_{Y}\right)$ be a closed complex manifold of complex dimension $m$, and $\mathcal{L} \rightarrow Y$ an ample line bundle which admits $m+1$ jointly transverse holomorphic sections. Let $\Omega_{\mathcal{L}}$ be a $J_{Y}$-Kähler representative of $c_{1}^{\mathcal{L}}$. If $n>m$ then $\left(\mathbb{C} P^{n} \times Y^{2 m}, \sigma \oplus \Omega_{\mathcal{L}}, J_{\mathbb{C} P^{n}} \oplus J_{Y}\right)$ admits a subcritical polarization of degree 1 .

Proof. The proof follows easily from Theorem 2.4.1: take $l=0$ (so that $E=0$ ) and $k=n+1$. Then $X_{E, k}=\mathbb{C} P^{n} \times Y$ and $\Omega_{E, k, \mathcal{L}}=\sigma \oplus \Omega_{\mathcal{L}}$.

We now turn to the

Proof of the statement in example 2.2.4. Consider the projective bundle $\mathbb{P}\left(E_{k, m}\right) \stackrel{\pi}{\rightarrow}$ $\mathbb{C} P^{m}$ where $E_{k, m}=\mathcal{O}_{\mathbb{C} P m}(-1) \oplus \mathbb{C}^{k}$.

We claim that there exists a biholomorphism $f: X_{k, m} \rightarrow \mathbb{P}\left(E_{k, m}\right)$ with the following properties:

- $f^{*} \mathcal{O}_{E_{k, m}}(1)=\mathcal{O}_{X_{k, m}}\left(H_{1}\right)$.

- $f^{*}\left(\pi^{*} \mathcal{O}_{\mathbb{C P}}(1)\right)=\mathcal{O}_{X_{k, m}}\left(H_{2}\right)$.

Indeed, write elements of $\mathbb{P}\left(E_{k, m}\right)$ as $(l,[z: w])$ where $l \in \mathbb{C} P^{m}, z \in l \subset \mathbb{C}^{m+1}$ and $w \in \mathbb{C}^{k}$. Consider the projection

$$
\rho: \mathbb{P}\left(E_{k, m}\right) \rightarrow \mathbb{C} P^{m+k}, \quad(l,[z: w]) \mapsto[z: w] .
$$

The preimage $\rho^{-1}([z: w])$ is a single point if $z \neq 0$ and a copy of $\mathbb{C} P^{m}$ if $z=0$. By uniqueness of blow-ups, this implies that $\mathbb{P}\left(E_{k, m}\right)$ is biholomorphic to the blow-up of $\mathbb{C} P^{m+k}$ along $P_{0}^{k-1}=\{[z: w] \mid z=0\}$. Under this identification, $H_{1}$ and $H_{2}$ correspond (up to linear equivalence) to the proper transforms of the hypersurfaces $\left\{w_{1}=0\right\}$ and $\left\{z_{0}=0\right\}$. Thus

$$
H_{1} \sim\left\{(l,[z: w]) \mid z \in l, w_{1}=0\right\}, \quad H_{2} \sim\left\{(l,[z: w]) \mid z \in l, z_{0}=0\right\},
$$

and the claim follows.

Note that the vector bundle $E_{k, m}$ is Griffiths semi-negative and $\mathcal{O}_{\mathbb{C} P m}(1)$ is ample. Moreover, $\mathcal{O}_{\mathbb{C P}}(1)$ admits $m+1$ jointly transverse sections. The corollary now follows from Theorem 2.4 .1 (with $l=1$ and $k>m$ ) by pulling everything back via $f$.

\subsection{Subcritical polarizations in low dimensions}

Surprisingly enough in real dimension $\leq 4$ there exist only two subcritical polarizations. 
Proposition 2.6.1. Let $\mathcal{P}=(M, \Omega, J ; \Sigma)$ be a subcritical polarization.

- If $\operatorname{dim}_{\mathbb{R}} M=2$ then $M=\mathbb{C} P^{1}, \Sigma=$ pt and $k_{\mathcal{P}}=1$.

- If $\operatorname{dim}_{\mathbb{R}} M=4$ then $M=\mathbb{C} P^{2}, \Sigma \cong \mathbb{C} P^{1}$ is a projective line and $k_{\mathcal{P}}=1$.

Proof. Assume first that $\operatorname{dim}_{\mathbb{R}} M=4$. Let $\Delta \subset M$ be the skeleton obtained from Corollary 3.4 below and denote by $g$ the genus of $\Sigma$. Being in dimension 4 , we shall identify 2-homology with 2-cohomology without explicitly mentioning Poincaré duality.

Since $\operatorname{dim}_{\mathbb{R}} \Delta<2$, by a general position argument we get that:

$$
H_{1}(M ; \mathbb{R})=H_{1}(\Sigma ; \mathbb{R}) \cong \mathbb{R}^{2 g}, \quad \text { and } \quad H_{2}(M ; \mathbb{R})=\mathbb{R}[\Sigma] .
$$

Thus $\chi(M)=3-4 g$ and $\sigma(M)=1$. As $b_{2}(M)=1$, there exists $a \in \mathbb{R}$ such that $c_{1}^{M}=a[\Omega]$. Substituting this into the signature formula $c_{1}^{M} \cdot c_{1}^{M}=2 \chi(M)+3 \sigma(M)$ we get $a^{2}[\Omega] \cdot[\Omega]=9-8 g$, which implies that $g \leq 1$. By the adjunction formula applied to $\Sigma$ we have:

$$
2 g-2=\Sigma \cdot \Sigma-c_{1}^{M} \cdot \Sigma=k_{\mathcal{P}}\left(k_{\mathcal{P}}-a\right)[\Omega] \cdot[\Omega] .
$$

As $g \leq 1$, we conclude that $a \geq k_{\mathcal{P}}>0$ and so $M$ is monotone. By the EnriquesKodaira classification of complex surfaces ([B-P-V]), a monotone Kähler surface $M$ must be rational. In particular it must have $\chi(M) \geq 3$ with equality if and only if $M=\mathbb{C} P^{2}$. As $\chi(M)=3-4 g$, this proves that $g=0$ and $M=\mathbb{C} P^{2}$.

Now the only smooth rational curves in $\mathbb{C} P^{2}$ are projective lines and conics. However, the complement of a conic in $\mathbb{C} P^{2}$ has the homotopy type of $\mathbb{R} P^{2}$ (see [Bi-1] for example), which contradicts subcriticality. Thus the only possibility we are left with is $M=\mathbb{C} P^{2}, \Sigma=$ projective line and $k_{\mathcal{P}}=1$.

Assume now that $\operatorname{dim}_{\mathbb{R}} M=2$. In this case $\Sigma$ is just a bunch of points. Subcriticality implies that $b_{1}(M \backslash \Sigma)=0$. However the only punctured surface with $b_{1}=0$ is the 2 -sphere minus one point.

Remark. J. Etnyre pointed out to us a more topological proof of Proposition 2.6.1 which does not use the Enriques-Kodaira classification.

\subsection{A remark regarding the explicitness of the examples}

Let $(M, \Omega, J)$ be a Kähler manifold with $[\Omega] \in H^{2}(M ; \mathbb{Z})$ and $\mathcal{L} \rightarrow M$ a holomorphic line bundle with $c_{1}^{\mathcal{L}}=k[\Omega]$. Let $|\mathcal{L}|=\mathbb{P}\left(H^{0}(M, \mathcal{L})\right)$ be the linear system defined by $\mathcal{L}$. In this paper we often make non-explicit statements of the kind "there exists $\Sigma \in|\mathcal{L}|$ such that the polarization $\mathcal{P}=(M, \Omega, J ; \Sigma)$ is subcritical" without specifying which $\Sigma$ precisely we take (It may be possible that for one choice of $\Sigma$ the corresponding polarization is subcritical while for another one it is not).

The justification is that the symplectomorphism type of $\left(\Sigma, \omega_{\Sigma}=\left.\Omega\right|_{\Sigma}\right)$ does not depend on the specific choice of $\Sigma \in|\mathcal{L}|$ (as long as it is smooth and reduced). 
Since what we are interested in is the symplectic topology of manifolds that can be realized as hypersurfaces in subcritical polarizations, we do not really care which "copy" of $\left(\Sigma, \omega_{\Sigma}\right)$ in $|\mathcal{L}|$ gives rise to the subcritical polarization. All we care about is that there exists at least one such element in $|\mathcal{L}|$.

Let us briefly explain why the symplectomorphism type of $\left(\Sigma, \omega_{\Sigma}\right)$ is uniquely determined by $\mathcal{L}$ itself. Suppose that there exists a smooth and reduced element $\Sigma \in|\mathcal{L}|$. In this case the subset $|\mathcal{L}|_{\text {smooth }} \subset|\mathcal{L}|$ consisting of all smooth and reduced divisors is the complement of a proper closed subvariety of $|\mathcal{L}|$. Therefore $|\mathcal{L}|_{\text {smooth }}$ is open dense in $|\mathcal{L}|$ and furthermore it is path connected. This implies that any two hypersurfaces $\Sigma^{\prime}, \Sigma^{\prime \prime} \in|\mathcal{L}|_{\text {smooth }}$ are isotopic inside $M$, in particular also diffeomorphic. Moreover, if we endow $\Sigma^{\prime}$ and $\Sigma^{\prime \prime}$ with the symplectic forms $\omega_{\Sigma^{\prime}}=\left.\Omega\right|_{\Sigma^{\prime}}$ and $\omega_{\Sigma^{\prime \prime}}=\left.\Omega\right|_{\Sigma^{\prime \prime}}$ respectively, then it easily follows by Moser's argument that $\left(\Sigma^{\prime}, \omega_{\Sigma^{\prime}}\right)$ is symplectomorphic to $\left(\Sigma^{\prime \prime}, \omega_{\Sigma^{\prime \prime}}\right)$.

\section{Decompositions of symplectic manifolds}

One of our main tools in studying the symplectic topology of subcritical manifolds is the decomposition technique developed in [Bi-1]. In this section we briefly summarize the ingredients of this theory which are relevant for our purposes. We refer the reader to [Bi-1] for more details.

Standard symplectic disc bundles. Let $\mathcal{P}=(M, \Omega, J ; \Sigma)$ be a polarization of degree $k_{\mathcal{P}}$ of a Kähler manifold.

Put $\omega_{\Sigma}=\left.\Omega\right|_{\Sigma}$ and let $\pi: N_{\Sigma} \rightarrow \Sigma$ be the (complex) normal line bundle of $\Sigma$ in $M$ with first Chern class $c_{1}^{N_{\Sigma}}=k_{\mathcal{P}}\left[\omega_{\Sigma}\right] \in H^{2}(\Sigma ; \mathbb{R})$. Let $\|\cdot\|$ be any hermitian metric on $N_{\Sigma}$ and denote by $E_{\Sigma}=\left\{v \in N_{\Sigma} \mid\|v\|<1\right\}$ the open unit disc bundle of $N_{\Sigma}$. Choose a connection $\nabla$ on $N_{\Sigma}$ with curvature $R^{\nabla}=2 \pi i k_{\mathcal{P}} \omega_{\Sigma}$ and denote by $\alpha^{\nabla}$ the associated transgression 1 -form on $N_{\Sigma} \backslash 0$ defined by:

- $\alpha_{(u)}^{\nabla}(u)=0, \alpha_{(u)}^{\nabla}(i u)=\frac{1}{2 \pi}$ for every $u \in N_{\Sigma} \backslash 0$.

- $\left.\alpha^{\nabla}\right|_{H^{\nabla}}=0$, where $H^{\nabla}$ is the horizontal distribution of $\nabla$.

With this normalization of $\alpha^{\nabla}$ we have $d \alpha^{\nabla}=-\pi^{*}\left(k_{\mathcal{P}} \omega_{\Sigma}\right)$. Define now the following symplectic form $\omega_{\text {can }}$ on $E_{\Sigma}$ :

$$
\omega_{\text {can }}=k_{\mathcal{P}} \pi^{*} \omega_{\Sigma}+d\left(r^{2} \alpha^{\nabla}\right)
$$

where $r$ is the radial coordinate along the fibres induced by $\|\cdot\|$. It is easy to check that $\omega_{\text {can }}$ is well defined, that it is symplectic and has the following three properties:

1. All fibres of $\pi: E_{\Sigma} \rightarrow \Sigma$ are symplectic with respect to $\omega_{\text {can }}$ and have area 1 .

2. The restriction of $\omega_{\text {can }}$ to the zero section $\Sigma \subset E_{\Sigma}$ equals to $k_{\mathcal{P}} \omega_{\Sigma}$.

3. $\omega_{\text {can }}$ is $S^{1}$-invariant with respect to the obvious circle action on $E_{\Sigma}$.

The subscript in $\omega_{\text {can }}$ suggests that this symplectic structure is canonical although the definition of $\left(E_{\Sigma}, \omega_{\text {can }}\right)$ a priori depends on $\|\cdot\|$ and $\nabla$. Indeed, different 
choices of $\|\cdot\|$ and $\nabla$ in fact lead to symplectically equivalent results. The following can be easily proved using a suitable version of Moser argument (see [M-S]):

Proposition 3.1. The symplectic type of $\left(E_{\Sigma}, \omega_{\text {can }}\right)$ depends only on the symplectic type of $\left(\Sigma, \omega_{\Sigma}\right)$. In fact, $\left(E_{\Sigma}, \omega_{\text {can }}\right)$ is uniquely characterized (up to symplectomorphism) by properties 1-3 above.

In view of the above proposition we shall henceforth call $\left(E_{\Sigma}, \omega_{\text {can }}\right)$ the standard symplectic disc bundle over $\left(\Sigma, \omega_{\Sigma}\right)$ modeled on $N_{\Sigma}$. Often we shall multiply $\omega_{\text {can }}$ by a positive number $c>0$ (usually by $\left.c=\frac{1}{k_{\mathcal{P}}}\right)$ and refer to $\left(E_{\Sigma}, c \omega_{\text {can }}\right.$ ) as the standard symplectic disc bundle with fibres of area $c^{2}$

Remarks. Here are two alternative descriptions of the symplectic manifold $\left(E_{\Sigma}, \omega_{\text {can }}\right)$ :

1. If we denote by $P_{\Sigma}=\left\{v \in N_{\Sigma} \mid\|v\|=1\right\} \subset N_{\Sigma}$ the unit circle bundle and by $D(1) \subset \mathbb{C}$ the open unit disc, then $\left(E_{\Sigma}, \omega_{\text {can }}\right) \cong\left(P_{\Sigma} \times_{S^{1}} D(1), k_{\mathcal{P}} \pi^{*} \omega_{\Sigma}+\right.$ $\left.d\left(r^{2} \alpha\right)\right)$, where $S^{1}$ acts diagonally in an obvious way on both components, $r$ is the radial coordinate on $D(1), \pi: P_{\Sigma} \rightarrow \Sigma$ is the projection and $\alpha$ is a connection 1-form on $P_{\Sigma}$ with $d \alpha=-k_{\mathcal{P}} \pi^{*} \omega_{\Sigma}$.

2. $\left(E_{\Sigma}, \omega_{\text {can }}\right)$ can be also viewed as a "one-sided" compactification of the negative symplectization of the contact manifold $\left(P_{\Sigma}, \xi=\operatorname{ker} \alpha\right)$. Indeed, it is not hard to see that $\left(E_{\Sigma} \backslash \Sigma, \omega_{\text {can }}\right)$ is symplectomorphic to the negative symplectization $\left(P_{\Sigma} \times(0, \infty), d\left(e^{-t} \alpha\right)\right)$ where $t$ is the coordinate on $(0, \infty)$.

Isotropic $C W$-complexes. Let $M$ be a smooth manifold. A subset $\Delta \subset M$ is called an embedded CW-complex if there exists an abstract $\mathrm{CW}$-complex $K$ and a homeomorphism $i: K \rightarrow \Delta \subset M$ such that for every cell $C \subset K$ the restriction $\left.i\right|_{\operatorname{Int} C \cong \operatorname{Int}\left(D^{\operatorname{dim} C}\right)}: \operatorname{Int} C \rightarrow M$ is a smooth embedding. We denote $\operatorname{dim} \Delta=$ $\operatorname{dim} K=\max \{\operatorname{dim} C \mid C \subset K$ is a cell of $K\}$. Henceforth we shall always assume all our $\mathrm{CW}$-complexes to be connected and finite.

Let $\Delta \subset(M, \Omega)$ be an embedded CW-complex in a symplectic manifold. If for every cell $C$ as above, $i(\operatorname{Int} C)$ is an isotropic submanifold of $(M, \Omega)$ we say that $\Delta$ is an isotropic $C W$-complex. Note that if $\Delta$ is isotropic we have $\operatorname{dim} \Delta \leq \frac{1}{2} \operatorname{dim}_{\mathbb{R}} M$. When this inequality is strict we call $\Delta$ a subcritical isotropic $C W$-complex.

The skeleton associated with a polarization. Let $\mathcal{P}=(M, \Omega, J ; \Sigma)$ be a polarized Kähler manifold. Let $\varphi_{\mathcal{P}}: M \backslash \Sigma \rightarrow \mathbb{R}$ be the associated function defined in Section 2. Denote by $g_{\Omega, J}=\Omega(\cdot, J \cdot)$ the Kähler Riemannian metric associated with the pair $(\Omega, J)$.

With this data fixed we have a distinguished subset $\Delta_{\mathcal{P}} \subset M \backslash \Sigma$ defined as follows. Consider the gradient vector field $\operatorname{grad}_{g_{\Omega, J}} \varphi_{\mathcal{P}}$ of the function $\varphi_{\mathcal{P}}$ with

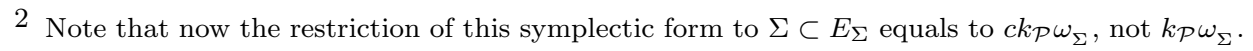


respect to the metric $g_{\Omega, J}$ and let $F_{t}$ be its flow. Define $\Delta_{\mathcal{P}} \subset M \backslash \Sigma$ to be the union of all the stable submanifolds corresponding to critical points of $\varphi_{\mathcal{P}}$, that is

$$
\Delta_{\mathcal{P}}=\left\{x \in M \backslash \Sigma \mid \lim _{t \rightarrow \infty} F_{t}(x) \in \operatorname{Crit}\left(\varphi_{\mathcal{P}}\right)\right\} .
$$

Note that $\Delta_{\mathcal{P}} \subset M \backslash \Sigma$ is compact since $F_{t}$ is complete at $-\infty$ and $\operatorname{Crit}\left(\varphi_{\mathcal{P}}\right)$ is a compact subset of $M \backslash \Sigma$.

We remark that $\Delta_{\mathcal{P}}$ is completely determined by the polarization $\mathcal{P}$ without any further choices since the function $\varphi_{\mathcal{P}}$ is determined (up to an additive constant) by $\mathcal{P}$. We shall therefore call $\Delta_{\mathcal{P}}$ the skeleton associated with the polarization $\mathcal{P}$.

The importance of $\Delta_{\mathcal{P}}$ lies in the following Theorem from [Bi-1]:

Theorem 3.2. Let $\mathcal{P}=(M, \Omega, J ; \Sigma)$ be a polarized Kähler manifold. Then the complement of the skeleton $\left(M \backslash \Delta_{\mathcal{P}}, \Omega\right)$ is symplectomorphic to the standard symplectic disc bundle $\left(E_{N_{\Sigma}}, \frac{1}{k_{\mathcal{P}}} \omega_{\text {can }}\right)$ over $\Sigma$ which is modeled on the normal bundle $N_{\Sigma}$, and has fibres of area $\frac{1}{k_{\mathcal{P}}}$.

It should be pointed out, however, that without any further assumptions on the function $\varphi_{\mathcal{P}}$ and the metric $g_{\Omega, J}$ the skeleton $\Delta_{\mathcal{P}}$ might have a very "wild" structure. Moreover, even if $\varphi_{\mathcal{P}}$ is Morse $\Delta_{\mathcal{P}}$ might be quite far from being a "reasonable" space (see [Bi-1] for further discussion on this issue). On the other hand, the following collection of results from [Bi-1] shows that it is always possible to modify $\Omega$ into $\Omega^{\prime}$ (which is diffeomorphic to $\Omega$ ) in such a way that the corresponding polarization $\mathcal{P}^{\prime}=\left(M, \Omega^{\prime}, J ; \Sigma\right)$ gives rise to a skeleton which is an isotropic CW-complex.

Theorem 3.3 ([Bi-1]). Let $\mathcal{P}=(M, \Omega, J ; \Sigma)$ be a subcritical polarization of degree $k_{\mathcal{P}}$. Then there exists a J-Kähler form $\Omega^{\prime}$ that coincides with $\Omega$ near $\Sigma$ and is cohomologous to $\Omega$ and such that the polarization $\mathcal{P}^{\prime}=\left(M, \Omega^{\prime}, J ; \Sigma\right)$ has the following properties:

1. The Stein manifold $\left(V=M \backslash \Sigma, J, \varphi_{\mathcal{P}^{\prime}}\right) \rightarrow \mathbb{R}$ is subcritical. Moreover, $\varphi_{\mathcal{P}^{\prime}}=\varphi_{\mathcal{P}}$ outside a compact subset of $V$ which contains all the critical points of $\varphi_{\mathcal{P}^{\prime}}$.

2. The skeleton $\Delta_{\mathcal{P}^{\prime}}$ associated with $\mathcal{P}^{\prime}$ is an $\Omega^{\prime}$-isotropic $C W$-complex with $\operatorname{dim}_{\mathbb{R}} \Delta_{\mathcal{P}^{\prime}}<\frac{1}{2} \operatorname{dim}_{\mathbb{R}} M$.

3. $\left(M \backslash \Delta_{\mathcal{P}^{\prime}}, \Omega^{\prime}\right)$ is symplectomorphic to the standard symplectic disc bundle $\left(E_{\Sigma}, \frac{1}{k_{\mathcal{P}}} \omega_{\text {can }}\right)$ modeled on the normal bundle $N_{\Sigma}$ of $\Sigma$ in $M$ and whose fibres have area $\frac{1}{k_{\mathcal{P}}}$. Moreover, a symplectomorphism $F_{\mathcal{P}}:\left(E_{\Sigma}, \frac{1}{k_{\mathcal{P}}} \omega_{\text {can }}\right) \rightarrow(M \backslash$ $\left.\Delta_{\mathcal{P}^{\prime}}, \Omega^{\prime}\right)$ can be chosen so that it sends the zero section $\Sigma \subset E_{\Sigma}$ onto $\Sigma \subset$ $M \backslash \Delta_{\mathcal{P}^{\prime}}$ identically, namely $\left.F_{\mathcal{P}}\right|_{\Sigma}=\mathbb{1}_{\Sigma}$.

For the proofs see Theorems 2.6.A, 2.6.C, and 8.2.A in [Bi-1].

The following is a simple consequence of Theorem 3.3 and Moser's argument: 
Corollary 3.4. Let $\mathcal{P}=(M, \Omega, J ; \Sigma)$ be a subcritical polarization of degree $k_{\mathcal{P}}$. Then there exists an $\Omega$-isotropic $C W$-complex $\Delta \subset M \backslash \Sigma$ with $\operatorname{dim} \Delta<\frac{1}{2} \operatorname{dim}_{\mathbb{R}} M$ and a symplectomorphism $F:\left(E_{\Sigma}, \frac{1}{k_{\mathcal{P}}} \omega_{\text {can }}\right) \rightarrow(M \backslash \Delta, \Omega)$ which sends the zero section $\Sigma \subset E_{\Sigma}$ onto $\Sigma \subset M \backslash \Delta$ identically.

\section{Lagrangian embeddings: theorems and examples}

\subsection{Setup}

Monotone manifolds. Recall that a symplectic manifold $(M, \Omega)$ is called monotone if there exists a positive real number $\lambda_{(M, \Omega)}$ such that $c_{1}^{M}=\lambda_{(M, \Omega)}[\Omega]$ in $H^{2}(M ; \mathbb{R})$. Similarly, $(M, \Omega)$ is called spherically monotone if the following two conditions are satisfied:

1. $c_{1}^{M}$ does not vanish on $\pi_{2}(M)$.

2. There exists a positive real number $\lambda$ such that $c_{1}^{M}=\lambda[\Omega]$ on $\pi_{2}(M)$.

Note that due to condition 1 the number $\lambda$ is uniquely determined by $\Omega$ hence we shall denote it from now on by $\lambda_{(M, \Omega)}$.

\section{Examples.}

1. $\left(\mathbb{C} P^{n}, \sigma_{\mathbb{C} P^{n}}\right)$ is monotone with $\lambda=n+1$.

2. $\left(\mathbb{C} P^{n} \times \mathbb{C} P^{m}, \sigma \oplus \sigma\right)$ is (spherically) monotone if and only if $n=m$.

3. Let $\left(Y, \omega_{Y}\right)$ be a closed symplectic manifold for which both $c_{1}^{Y}$ and $\omega_{Y}$ vanish on $\pi_{2}(Y)$. Then $\left(\mathbb{C} P^{n} \times Y, \sigma \oplus \omega_{Y}\right)$ is spherically monotone but in general not monotone. Again, $\lambda=n+1$.

Tame symplectic manifolds. In what follows we call a symplectic manifolds $\left(X, \omega_{X}\right)$ tame if it admits an $\omega_{X}$-compatible almost complex structure $J_{X}$ such that $\left(X, \omega_{X}, J_{X}\right)$ is either geometrically bounded in the sense of $[\mathrm{A}-\mathrm{L}-\mathrm{P}]$ or convex at infinity in the sense of [E-G]. This class of manifolds includes: closed symplectic manifolds, Stein manifolds / domains, and interiors of compact symplectic manifolds with $J_{X}$-convex boundary. We remark that $X$ is allowed to be zero dimensional (namely a point).

\subsection{Main results}

Let $\left(\Sigma, \omega_{\Sigma}\right)$ be a closed symplectic manifold. We say that it can be realized as a hypersurface of a subcritical polarization if it can be embedded into a subcritical polarization $\mathcal{P}=(M, \Omega, J ; \Sigma)$ in such a way that $\omega_{\Sigma}=\left.\Omega\right|_{\Sigma}$.

Theorem 4.2.1. Let $\left(\Sigma, \omega_{\Sigma}\right)$ be a symplectic manifold (with $\operatorname{dim} \Sigma>0$ ) that can be realized as a hypersurface of a subcritical polarization of degree $k_{\mathcal{P}}$. Let $\left(X, \omega_{X}\right)$ be a tame symplectic manifold. Assume that the following conditions are satisfied: 
1. $\left[\omega_{X}\right]$ and $c_{1}^{X}$ both vanish on $\pi_{2}(X)$ (resp. in $H^{2}(X ; \mathbb{R})$ ).

2. $\left(\Sigma, \omega_{\Sigma}\right)$ is spherically monotone (resp. monotone).

3. $\lambda_{\left(\Sigma, \omega_{\Sigma}\right)}$ and $k_{\mathcal{P}}$ satisfy the following inequality:

$$
\frac{\lambda_{\left(\Sigma, \omega_{\Sigma}\right)}}{k_{\mathcal{P}}}>\frac{\operatorname{dim}_{\mathbb{C}} \Sigma+\operatorname{dim}_{\mathbb{C}} X+1}{2} .
$$

Then $\left(\Sigma \times X, \omega_{\Sigma} \oplus \omega_{X}\right)$ has no closed simply connected Lagrangian submanifolds (resp. closed Lagrangian submanifolds $L$ with $H_{1}(L ; \mathbb{Z})=0$ ).

Remark. Note that the assumption " $\left[\omega_{X}\right]$ vanishes on $\pi_{2}(X)$ " allows $X$ to be a closed manifold (e.g. $\left.T^{2 m}\right)$. However, the analogous condition " $\left[\omega_{X}\right]$ vanishes in $H^{2}(X, \mathbb{R})$ " implies that $X$ cannot be a closed manifold (unless it is 0 -dimensional).

Strongly monotone submanifolds. Let $\left(\Sigma, \omega_{\Sigma}\right)$ be a symplectic manifold that can be realized as a hypersurface of a polarization $\mathcal{P}=(M, \Omega, J ; \Sigma)$. We say that $\left(\Sigma, \omega_{\Sigma}\right)$ is strongly monotone in $\mathcal{P}$ if there exist $\eta, \nu \in \mathbb{N}$ such that

$$
\eta c_{1}^{\Sigma}=\nu c_{1}^{N_{\Sigma}} \quad \text { in } \quad H^{2}(\Sigma ; \mathbb{Z}) .
$$

Here $N_{\Sigma} \rightarrow \Sigma$ stands for the (complex) normal line bundle of $\Sigma$ in $M$.

We remark that the integers $\eta, \nu$ depend on the polarization $\mathcal{P}$ and not only on $\left(\Sigma, \omega_{\Sigma}\right)$ because we need to know the value of $c_{1}^{N_{\Sigma}}$ in $H^{2}(\Sigma ; \mathbb{Z})$ (not just in $\left.H^{2}(\Sigma ; \mathbb{R})\right)$, or in other words, we need to know the topological type of $N_{\Sigma}$. The ratio $\frac{\nu}{\eta}$ however depends only on $\left(\Sigma, \omega_{\Sigma}\right)$ and the degree of the polarization $k_{\mathcal{P}}$ since it is equal to $\frac{\lambda_{\left(\Sigma, \omega_{\Sigma}\right)}}{k_{\mathcal{P}}}\left(\right.$ where $c_{1}^{\Sigma}=\lambda_{\left(\Sigma, \omega_{\Sigma}\right)}\left[\omega_{\Sigma}\right]$ in $\left.H^{2}(\Sigma ; \mathbb{R})\right)$.

Theorem 4.2.2. Let $\left(\Sigma, \omega_{\Sigma}\right)$ be a symplectic manifold (with $\operatorname{dim} \Sigma>0$ ) that can be realized as a strongly monotone hypersurface of a subcritical polarization $\mathcal{P}$ with $\eta, \nu$ defined as above. Let $\left(X, \omega_{X}\right)$ be a tame symplectic manifold. Assume that the following conditions are satisfied:

1. $\left[\omega_{X}\right]$ vanishes in $H^{2}(X ; \mathbb{R})$ and $c_{1}^{X}$ is a torsion element in $H^{2}(X ; \mathbb{Z})$. Denote by $t_{X} \in \mathbb{N}$ its order.

2. $\eta, \nu$ satisfy the inequality:

$$
\frac{\nu}{\eta}=\frac{\lambda_{\left(\Sigma, \omega_{\Sigma}\right)}}{k_{\mathcal{P}}}>\frac{\operatorname{dim}_{\mathbb{C}} \Sigma+\operatorname{dim}_{\mathbb{C}} X+1}{2} .
$$

Let $L \subset\left(\Sigma \times X, \omega_{\Sigma} \oplus \omega_{X}\right)$ be a closed Lagrangian submanifold. Then $H^{1}(L ; \mathbb{Z} / q \mathbb{Z})$ $\neq 0$, where $q=\nu \frac{\operatorname{lcm}\left(2, \eta, t_{X}\right)}{\eta} \in \mathbb{N}$.

Remark. By the universal coefficient formula, " $H^{1}(L ; \mathbb{Z} / q \mathbb{Z}) \neq 0$ " is equivalent to saying that $H_{1}(L ; \mathbb{Z})$ either has a non-trivial free summand, or it contains a non-trivial finite cyclic subgroup whose order divides $q$. 
Theorem 4.2.3. Let $\left(\Sigma, \omega_{\Sigma}\right)$ and $\left(X, \omega_{X}\right)$ be symplectic manifolds satisfying the same assumptions as in Theorem 4.2.2, with the exception that $\left[\omega_{X}\right]$ is only assumed to vanish on $\pi_{2}(X)$. Let $L \subset\left(\Sigma \times X, \omega_{\Sigma} \oplus \omega_{X}\right)$ be a closed Lagrangian submanifold. Then either $\pi_{1}(L)$ contains an infinite cyclic subgroup, or $H_{1}(L ; \mathbb{Z})$ contains a non-trivial finite cyclic subgroup whose order divides $q$, where $q$ is defined in Theorem 4.2.2.

\subsection{Corollaries and examples}

As a corollary to Theorem 4.2.1 we have:

Corollary 4.3.1. Let $\left(X^{2 m}, \omega_{X}\right)$ be a $2 m$-dimensional tame symplectic manifold, and suppose that $c_{1}^{X}$ and $\omega_{X}$ both vanish on $\pi_{2}(X)$ (resp. in $H^{2}(X ; \mathbb{R})$ ). Let $n \geq m$. Then $\left(\mathbb{C} P^{n} \times X^{2 m}, \sigma \oplus \omega_{X}\right)$ has no closed simply connected Lagrangian submanifolds (resp. closed Lagrangian submanifolds $L$ with $H_{1}(L ; \mathbb{Z})=0$ ).

Proof. In Section 2 we have seen that $\left(\mathbb{C} P^{n}, \sigma\right)$ can be realized as a hypersurface of a subcritical polarization (of $\mathbb{C} P^{n+1}$ ) of degree 1 . Note that $\left(\mathbb{C} P^{n}, \sigma\right)$ is monotone with $\lambda_{\left(\mathbb{C} P^{n}, \sigma\right)}=n+1$. Therefore the inequality

$$
\frac{\lambda_{\left(\Sigma, \omega_{\Sigma}\right)}}{k_{\mathcal{P}}}>\frac{\operatorname{dim}_{\mathbb{C}} \Sigma+\operatorname{dim}_{\mathbb{C}} X+1}{2} .
$$

becomes $n+1>\frac{1}{2}(n+m+1)$ which is equivalent to $n \geq m$.

Construction. Note that the result is sharp in the following sense. Let $m=n+1$. Then for any $2 m$-dimensional symplectic manifold $\left(X^{2 m}, \omega_{X}\right)$, there exists $a_{0}>0$ such that for every $a \geq a_{0},\left(\mathbb{C} P^{n} \times X^{2 m}, \sigma \oplus a \omega_{X}\right)$, admits a Lagrangian $2 n+1$ sphere.

The construction goes as follows. Denote by $S^{2 n+1} \subset \mathbb{C}^{n+1}$ the unit sphere and by $h: S^{2 n+1} \rightarrow \mathbb{C} P^{n}$ the Hopf map. Then

$$
S^{2 n+1} \ni z \mapsto(\bar{z}, h(z)) \in\left(\mathbb{C}^{n+1} \times \mathbb{C} P^{n}, \omega_{\text {std }} \oplus \sigma\right)
$$

is a Lagrangian embedding (see $[\mathrm{A}-\mathrm{L}-\mathrm{P}]$ ).

Now let $\left(X, \omega_{X}\right)$ be a $2 n+2$-dimensional symplectic manifold that admits a symplectic embedding of a closed $2 n+2$-dimensional ball of radius 1 , say $\varphi$ : $B^{2 n+1}(1) \rightarrow\left(X, \omega_{X}\right)$. It is easy to check that

$$
S^{2 n+1}=\partial B^{2 n+2}(1) \ni z \mapsto(\varphi(\bar{z}), h(z)) \in\left(X \times \mathbb{C} P^{n}, \omega_{X} \oplus \sigma_{\text {std }}\right)
$$

is a Lagrangian embedding.

To complete the construction, note that by Darboux' Theorem for every symplectic manifold $\left(X, \omega_{X}\right)$ there exists $a_{0}>0$ such that for every $a \geq a_{0},\left(X, a \omega_{X}\right)$ admits an embedding of a ball as above. 
Examples of $\left(X^{2 m}, \omega_{X}\right)$. Let us mention a few examples of manifolds that can play the role of $\left(X^{2 m}, \omega_{X}\right)$ in Theorem 4.2.1 and Corollary 4.3.1:

- $X=\mathrm{pt}$.

- Any product of closed orientable surfaces of genus $\geq 1$ (e.g. $T^{2 m}$ ), the product being endowed with any symplectic structure. More generally, any closed aspherical symplectic manifold.

- Any Stein manifold $\left(X, \omega_{X}\right)$ with $c_{1}^{X}$ vanishing on $\pi_{2}(X)\left(\right.$ resp. in $\left.H^{2}(X ; \mathbb{R})\right)$. For example, $\left(\mathbb{C}^{m}, \omega_{\text {std }}\right)$ or cotangent bundles $X=T^{*}(N)$ of any closed manifold $N$, endowed with their standard symplectic structure.

- Products of manifolds from the above list.

Proof of Theorem A. Immediate from Corollary 4.3.1.

For symplectic manifolds $\left(X, \omega_{X}\right)$ with $c_{1}^{X}$ being a torsion element in $H^{2}(X ; \mathbb{Z})$ we get from Theorem 4.2.2:

Corollary 4.3.2. Let $\left(X^{2 m}, \omega_{X}\right)$ be a $2 m$-dimensional exact tame symplectic manifold, and suppose that $c_{1}^{X}$ is a torsion element of order $t_{X}$ in $H^{2}(X ; \mathbb{Z})$. In case $t_{X}$ is even put $\tau_{X}=\frac{1}{2} t_{X}$, in case $t_{X}$ is odd put $\tau_{X}=t_{X}$. Let $n \geq m$. Then every closed Lagrangian submanifold $L \subset\left(\mathbb{C} P^{n} \times X^{2 m}, \sigma \oplus \omega_{X}\right)$ must satisfy $H^{1}\left(L ; \mathbb{Z} /(2 n+2) \tau_{X} \mathbb{Z}\right) \neq 0$.

Proof. Note that $\left(\mathbb{C} P^{n}, \sigma\right)$ is strongly monotone in $\mathbb{C} P^{n+1}$ with $\eta=1$ and $\nu=n+1$. Hence by Theorem $4.2 .2, H^{1}(L ; \mathbb{Z} / q \mathbb{Z}) \neq 0$ for $q=(n+1) \operatorname{lcm}\left(2, t_{X}\right)=2(n+1) \tau_{X}$.

Using Theorem 4.2.3 instead of Theorem 4.2.2, the assumption on the exactness of $\omega_{X}$ can be weakened (so as to allow $X$ to be a closed manifold):

Corollary 4.3.3. Let $\left(X^{2 m}, \omega_{X}\right), t_{X}, \tau_{X}$ and $n \geq m$ be as in Corollary 4.3.2, with the exception that $\left[\omega_{X}\right]$ vanishes on $\pi_{2}(X)$ instead of the assumption that $\omega_{X}$ is exact. Let $L \subset\left(\mathbb{C} P^{n} \times X^{2 m}, \sigma \oplus \omega_{X}\right)$ be a closed Lagrangian submanifold. Then either $\pi_{1}(L)$ has an infinite cyclic subgroup, or $H_{1}(L ; \mathbb{Z})$ contains a non-trivial finite cyclic subgroup whose order divides $(2 n+2) \tau_{X}$.

Proof of Theorem B. Immediate from Corollaries 4.3.2 and 4.3.3, with $\tau_{X}=1$.

Another class of examples. Let us describe another class of examples to which Theorem 4.2.1 applies. Let $\left(Y^{2 k}, \omega_{Y}, J_{Y}\right)$ be a Kähler manifold of real dimension $2 k$ and let $\mathcal{L}_{Y} \rightarrow Y$ be a holomorphic line bundle with $c_{1}^{\mathcal{L}_{Y}}=\left[\omega_{Y}\right]$. Assume that:

1. $c_{1}^{Y}$ and $\omega_{Y}$ both vanish on $\pi_{2}(Y)$.

2. The line bundle $\mathcal{L}_{Y} \rightarrow Y$ is very ample (or more generally, $\mathcal{L}_{Y}$ admits $k+1$ jointly transverse holomorphic sections, see Section 2). 
Consider the line bundle $\mathcal{L}=\pi_{\mathbb{C} P^{n}}^{*} \mathcal{O}(1) \otimes \pi_{Y}^{*} \mathcal{L}_{Y}$ on $\mathbb{C} P^{n} \times Y$. Here, $\pi_{\mathbb{C} P^{n}}$ : $\mathbb{C} P^{n} \times Y \rightarrow \mathbb{C} P^{n}$ and $\pi_{Y}: \mathbb{C} P^{n} \times Y \rightarrow Y$ are the obvious projections. Finally, let $\Sigma^{2 n+2 k-2} \subset \mathbb{C} P^{n} \times Y^{2 k}$ be the zero set of a generic holomorphic section of the line bundle $\mathcal{L}$. It follows from Bertini's theorem that $\Sigma$ is irreducible, smooth and reduced. We denote by $\omega_{\Sigma}$ the restriction $\left.\left(\sigma \oplus \omega_{Y}\right)\right|_{\Sigma}$. Note that the symplectic type of $\left(\Sigma, \omega_{\Sigma}\right)$ does not depend on the choice of the generic section used to define $\Sigma$ (see the discussion in Section 2.7 above).

Corollary 4.3.4. Let $\left(Y^{2 k}, \omega_{Y}\right)$ and $\Sigma$ be as above, and let $\left(X^{2 m}, \omega_{X}\right)$ be a $2 m$ dimensional tame symplectic manifold such that $c_{1}^{X}$ and $\omega_{X}$ both vanish on $\pi_{2}(X)$. Assume that $n>k+m$. Then $\left(\Sigma \times X, \omega_{\Sigma} \oplus \omega_{X}\right)$ has no simply connected closed Lagrangian submanifolds.

Proof. By Corollary 2.5.1, $\left(\mathbb{C} P^{n} \times Y, \sigma \oplus \omega_{Y}, J_{\mathbb{C} P^{n}} \oplus J_{Y} ; \Sigma\right)$ is a subcritical polarization of degree 1. By Lemma 5.1.2 the vanishing of $c_{1}^{Y}$ and $\omega_{Y}$ on $\pi_{2}(Y)$ implies that $\left(\Sigma, \omega_{\Sigma}\right)$ is spherically monotone with $\lambda_{\left(\Sigma, \omega_{\Sigma}\right)}=n$. Hence by Theorem 4.2.1, there exists no simply connected closed Lagrangian submanifold provided that $n>\frac{(n+k-1)+m+1}{2}$, i.e. $n>k+m$.

Again, $\left(X, \omega_{X}\right)$ can be taken to be any of the manifolds in the list mentioned after Corollary 4.3.1. As for $\left(Y, \omega_{Y}, J_{Y}\right)$, one can take $\left(Y, J_{Y}\right)$ to be an Abelian variety endowed with an ample line bundle $\mathcal{L}_{Y}$ whose order of divisibility in $\operatorname{Pic}\left(Y, J_{Y}\right)$ is at least 3 . It is well known that such a line bundle is very ample (see $[\mathrm{G}-\mathrm{H}]$ ). For $\omega_{Y}$ one can take any Kähler representative of $c_{1}^{\mathcal{L}_{Y}}$.

Proof of Theorem E. Apply Corollary 4.3.4 with $Y=C \subset \mathbb{C} P^{n}$ and $\mathcal{L}_{Y} \rightarrow Y$ the pullback of the hyperplane bundle on $\mathbb{C} P^{n}$. The assumption genus $(C)>0$ ensures $\pi_{2}(Y)=0$. The explicit form of $\Sigma$ follows from Example 2.2.2.

\section{Lagrangian embeddings: proofs}

In this section we prove our main results on Lagrangian embeddings: Theorems 4.2.1, 4.2.2, 4.2.3, and Theorem $\mathrm{C}$ from the introduction.

\subsection{Preparation for the proofs}

The following theorem from $[\mathrm{B}-\mathrm{C}]$ is an important ingredient in our study of Lagrangian submanifolds. A special case of this theorem, namely when $V=\mathbb{C}^{n}$, has been established before by Polterovich [Po], in [A-L-P] and by $\mathrm{Oh}[\mathrm{Oh}-2]$. See also Viterbo [Vi-1] for related results)

A Lagrangian submanifold $L \subset(M, \omega)$ is called monotone if $[\omega]=\lambda \mu$ on 
$\pi_{2}(M, L)$ for some constant $\lambda>0$ (cf. [Oh-1]). Here $\mu: \pi_{2}(M, L) \rightarrow \mathbb{Z}$ denotes the Maslov index. Note that if $(M, \omega)$ admits a monotone Lagrangian submanifold then $(M, \omega)$ is automatically spherically monotone.

Theorem 5.1.1 ([B-C]). Let $\left(V, J_{V}, \varphi\right)$ be a subcritical Stein manifold and $\left(X, \omega_{X}, J_{X}\right)$ a tame symplectic manifold for which $\omega_{X}$ vanishes on $\pi_{2}(X)$. Let $L \subset\left(V \times X, \omega_{\varphi} \oplus \omega_{X}\right)$ be a closed Lagrangian submanifold. Then there exists a non-constant $J_{V} \oplus J_{X}$-holomorphic disc $D \subset V \times X$ with $\partial D \subset L$ such that $\mu([D]) \leq \operatorname{dim} L+1$. Moreover, if we assume that $L$ is monotone, $H^{1}(L ; \mathbb{Z} / 2 \mathbb{Z}) \neq 0$ and that $\operatorname{dim}_{\mathbb{R}}(V \times X) \geq 4$, then the disc $D$ may be assumed to satisfy $\mu([D]) \leq$ $\operatorname{dim} L$.

We shall also need the following lemma.

Lemma 5.1.2. Let $\mathcal{P}=(M, \Omega, J ; \Sigma)$ be a polarization of degree $k_{\mathcal{P}}$. Assume that either $\operatorname{dim}_{\mathbb{R}} M \geq 6$, or $\operatorname{dim}_{\mathbb{R}} M=4$ and $\mathcal{P}$ is subcritical. Then:

1. $\Omega$ vanishes on $\pi_{2}(M)$ if and only if $\omega_{\Sigma}$ vanishes on $\pi_{2}(\Sigma)$.

2. If $\left(\Sigma, \omega_{\Sigma}\right)$ is spherically monotone (resp. monotone) then so is also $(M, \Omega)$. Furthermore, in this case $\lambda_{(M, \Omega)}=\lambda_{\left(\Sigma, \omega_{\Sigma}\right)}+k_{\mathcal{P}}$.

Proof. If $\operatorname{dim}_{\mathbb{R}} M \geq 6$ the lemma follows immediately from Lefschetz hyperplane theorem, which implies that the inclusions $\Sigma \hookrightarrow M$ induces surjective maps on $\pi_{2}$ and $H_{2}$. The case of subcritical $\mathcal{P}$ with $\operatorname{dim}_{\mathbb{R}} M=4$ follows from Corollary 3.4 and a general position argument. The formula $\lambda_{(M, \Omega)}=\lambda_{\left(\Sigma, \omega_{\Sigma}\right)}+k_{\mathcal{P}}$ is an immediate consequence of the adjunction formula (see $[\mathrm{G}-\mathrm{H}])$.

\subsection{Proof of Theorem 4.2.1}

Outline of the proof. Let us explain first the main ideas of the proof and how the fact that $\left(\Sigma, \omega_{\Sigma}\right)$ can be realized as a hypersurface of a subcritical polarization is used.

Suppose, contrary to the statement of the theorem, that $\left(\Sigma \times X, \omega_{\Sigma} \oplus \omega_{X}\right)$ does admit a simply connected Lagrangian submanifold $L$.

By assumption there exists a subcritical polarization $\mathcal{P}=(M, \Omega, J ; \Sigma)$ with $\left.\Omega\right|_{\Sigma}=\omega_{\Sigma}$. The first step of the proof is to climb one dimension higher and construct a monotone Lagrangian submanifold $L_{r} \subset\left((M \backslash \Sigma) \times X, \Omega \oplus \omega_{X}\right)$ which is built as a circle bundle over $L$. For this purpose we use the decomposition technique described in Section 3.

The next step relies on the fact that $M \backslash \Sigma$ is a subcritical Stein manifold. By Theorem 5.1.1 there exists a non-constant holomorphic disc $D \subset(M \backslash \Sigma) \times X$ with boundary on $L_{r}$ such that:

$$
\mu([D]) \leq \operatorname{dim}_{\mathbb{C}}(M \times X) .
$$


Next we combine the disc bundle structure on $M \backslash \Delta$, described in Section 3, with the fact that $L$ is simply connected to cup the disc $D$ with another symplectic disc so as to obtain a sphere $S \subset M \times X$ with $\int_{S} \Omega>0$. Moreover, we can compute the value of $\int_{S} \Omega$.

The upper bound on $\mu([D])$ and a computation of the Maslov number of the other disc give us an upper bound on the first Chern number $c_{1}([S])$ in terms of $\operatorname{dim}_{\mathbb{C}}(M \times X)$.

Finally we use the monotonicity of $(M, \Omega)$. Comparing the ratio between the upper bound on $c_{1}([S])$ and the area $\int_{S} \Omega$ with the number $\lambda_{(M, \Omega)}$ will give us a contradiction. It turns out that the inequality in assumption 3 of the statement of Theorem 4.2.1 is precisely the one needed in order to get this contradiction.

We now turn to the

Proof of Theorem 4.2.1. We first prove the statement of the theorem regarding simply connected Lagrangians. The case of Lagrangians with $H_{1}(L ; \mathbb{Z})=0$ is similar and we will indicate at the end of the proof the needed adjustments.

Suppose that there exists a simply connected Lagrangian $L \subset\left(\Sigma \times X, \omega_{\Sigma} \oplus \omega_{X}\right)$. By assumption, $\Sigma$ can be included in a subcritical polarization $\mathcal{P}=(M, \Omega, J ; \Sigma)$ with $\left.\Omega\right|_{\Sigma}=\omega_{\Sigma}$.

Step 1. Let $\left(E_{\Sigma}, \omega_{\text {can }}\right)$ be the standard symplectic disc bundle over $\left(\Sigma, \omega_{\Sigma}\right)$ modeled on the normal line bundle $N_{\Sigma}$ of $\Sigma$ in $M$ and denote by $\pi: E_{\Sigma} \rightarrow \Sigma$ the projection.

For every $0<r<1$ write

$$
P_{r}=\left\{v \in E_{\Sigma} \mid\|v\|=r\right\} \subset E_{\Sigma}
$$

for the circle subbundle of radius $r$. Denote by $\hat{\pi}_{r}: P_{r} \times X \rightarrow \Sigma \times X$ the obvious projection coming from $P_{r} \rightarrow \Sigma$. Next, write $L_{r} \subset P_{r} \times X \subset E_{\Sigma} \times X$ for the restriction of the circle bundle $P_{r} \times X \rightarrow \Sigma \times X$ to $L$, namely $L_{r}=\hat{\pi}_{r}^{-1}(L)$. Finally, for every fixed $0<r<1$ we endow $E_{\Sigma} \times X$ with the following symplectic form

$$
\hat{\omega}_{r}=\frac{1}{k_{\mathcal{P}}} \omega_{\text {can }} \oplus\left(1-r^{2}\right) \omega_{X} .
$$

A straightforward computation (based on the definition of $\omega_{\text {can }}$ ) shows that $L_{r} \subset\left(E_{\Sigma} \times X, \hat{\omega}_{r}\right)$ is a Lagrangian submanifold.

We now have the following

Lemma 5.2.1. The circle bundle $\left.\hat{\pi}_{r}\right|_{L_{r}}: L_{r} \rightarrow L$ is trivial. In particular $L_{r} \cong$ $L \times S^{1}$.

The proof is given after the end of the present proof.

Note that the fibres of the circle bundle $L_{r} \rightarrow L$ inherit an orientation induced from the circle action on the fibres of $P_{r} \rightarrow \Sigma$. In what follows we shall denote 
by $\gamma$ a fibre of the circle bundle $L_{r} \rightarrow L$ endowed with this orientation. With this notation we have $\pi_{1}\left(L_{r}\right)=\mathbb{Z}[\gamma]$.

Step 2. Let $\varphi_{\mathcal{P}}: V=M \backslash \Sigma \rightarrow \mathbb{R}$ be the plurisubharmonic function on the Stein manifold $(V, J)$ associated with $\mathcal{P}$. Let $\Delta_{\mathcal{P}}$ be the corresponding skeleton.

Due to Theorem 3.3 we may assume (replacing $\Omega$ with $\Omega^{\prime}$ ) that $\left(V, J, \varphi_{\mathcal{P}}\right)$ is a subcritical Stein manifold, that $\Delta_{\mathcal{P}}$ is a subcritical isotropic CW-complex, and that there exist a symplectomorphism

$$
F_{\mathcal{P}}:\left(E_{\Sigma}, \frac{1}{k_{\mathcal{P}}} \omega_{\text {can }}\right) \rightarrow\left(M \backslash \Delta_{\mathcal{P}}, \Omega\right)
$$

which sends the zero section $\Sigma \subset E_{\Sigma}$ identically onto $\Sigma \subset M$.

Denote by $G$ the symplectomorphism

$$
G=F_{\mathcal{P}} \times \mathbb{1}:\left(E_{\Sigma} \times X, \hat{\omega}_{r}\right) \rightarrow\left(\left(M \backslash \Delta_{\mathcal{P}}\right) \times X, \widehat{\Omega}_{r}\right),
$$

where $\widehat{\Omega}_{r}=\Omega \oplus\left(1-r^{2}\right) \omega_{X}$.

Consider now the Lagrangian submanifold $G\left(L_{r}\right) \subset\left(M \times X, \widehat{\Omega}_{r}\right)$. Note that $G\left(L_{r}\right)$ lies in fact inside $\left(V \times X, \omega_{\varphi_{\mathcal{P}}} \oplus\left(1-r^{2}\right) \omega_{X}\right)$.

Lemma 5.2.2. There exists $0<r<1$ such that $G\left(L_{r}\right) \subset\left(V \times X, \omega_{\varphi_{\mathcal{P}}} \oplus\left(1-r^{2}\right) \omega_{X}\right)$ is monotone.

Again, the proof is postponed until the end of the current proof. From now on, let $r$ be the constant provided by Lemma 5.2.2.

Step 3. Let $J_{X}$ be an $\omega_{X}$-compatible almost complex structure which realizes $\left(X, \omega_{X}, J_{X}\right)$ as a tame symplectic manifold. Put $J_{V \times X}=J \oplus J_{X}$.

We are now in the position to apply Theorem 5.1 .1 since $\left(V, J, \omega_{\varphi_{\mathcal{P}}}\right)$ is a subcritical Stein manifold, $\omega_{X}$ vanishes on $\pi_{2}(X), G\left(L_{r}\right)$ is monotone, $H^{1}\left(L_{r} ; \mathbb{Z}_{2}\right) \neq 0$, and $\operatorname{dim}_{\mathbb{R}}(V \times X) \geq \operatorname{dim}_{\mathbb{R}} V \geq 4$.

By Theorem 5.1.1 there exists a non-constant $J_{V \times X}$-holomorphic disc $\widetilde{D} \subset$ $V \times X$ with boundary on $G\left(L_{r}\right)$ such that $\mu([\widetilde{D}]) \leq \operatorname{dim}_{\mathbb{C}} V+\operatorname{dim}_{\mathbb{C}} X$.

Since $\operatorname{dim}_{\mathbb{R}} V \geq 4$, the (real) codimension of $\Delta_{\mathcal{P}} \times X$ in $V \times X$ is higher than 2. So by slightly perturbing $\widetilde{D}$ away from its boundary we get a symplectic disc (which we continue to denote by $\widetilde{D})$ with $\int_{\widetilde{D}} \widehat{\Omega}_{r}>0$ and $\mu([\widetilde{D}]) \leq \operatorname{dim}_{\mathbb{C}} V+\operatorname{dim}_{\mathbb{C}} X$ that lies in the complement of $\Delta_{\mathcal{P}} \times X$ in $V \times X$. In other words, we may assume $\widetilde{D}$ to lie in the image of $\left(E_{\Sigma} \backslash \Sigma\right) \times X$ under $G$. Going back to $E_{\Sigma} \times X$ via $G^{-1}$ we get a symplectic disc

$$
D=G^{-1}(\widetilde{D}) \subset\left(E_{\Sigma} \backslash \Sigma\right) \times X
$$

with boundary on $L_{r}$ such that $\int_{D} \hat{\omega}_{r}>0$ and $\mu([D]) \leq \operatorname{dim}_{\mathbb{C}} \Sigma+\operatorname{dim}_{\mathbb{C}} X+1$.

Step 4. As $\pi_{1}\left(L_{r}\right)=\mathbb{Z}[\gamma]$, there exists a unique integer $l$ such that $\partial[D]=l[\gamma]$ in $\pi_{1}\left(L_{r}\right)$. We claim that $l<0$. 
To prove this, denote by $\operatorname{pr}_{X}: E_{\Sigma} \times X \rightarrow X$ and $\operatorname{pr}_{E_{\Sigma}}: E_{\Sigma} \times X \rightarrow E_{\Sigma}$ the obvious projections. Let $\overline{E_{\Sigma}(r)}=\left\{v \in E_{\Sigma} \mid\|v\| \leq r\right\} \rightarrow \Sigma$ be the closed disc subbundle of radius $r$ in $E_{\Sigma}$ and consider the pull-back disc bundle

$$
\pi \times \mathbb{1}: \overline{E_{\Sigma}(r)} \times X \rightarrow \Sigma \times X .
$$

Pick a point $p \in L$ and denote by $F_{r}=(\pi \times \mathbb{1})^{-1}(p)$ the fibre over $p$. Note that $F_{r}$ is a symplectic disc (with respect to $\hat{\omega}_{r}$ ) with boundary on $L_{r}$. Moreover, with the orientation induced on $F_{r}$ by $\hat{\omega}_{r}$ we obviously have $\partial\left[F_{r}\right]=[\gamma] \in \pi_{1}\left(L_{r}\right)$.

As $\partial[D]=l[\gamma]$, we can choose another representative $D^{\prime}$ of $[D] \in \pi_{2}\left(E_{\Sigma} \times X, L_{r}\right)$ whose boundary winds around one of the fibres $l$ times, that is, $\partial D^{\prime}=l \gamma$.

Consider now the sphere $S=D^{\prime} \cup_{\partial D^{\prime}}(-l) F_{r}$ obtained from gluing the disc $D^{\prime}$ along the boundary to the $(-l)$ multiple cover of the fibre $F_{r}$. Since $\omega_{X}$ vanishes on $\pi_{2}(X)$ and $\operatorname{pr}_{X}\left(F_{r}\right)=\mathrm{pt}$ we have

$$
\int_{D^{\prime}} \operatorname{pr}_{X}^{*} \omega_{X}=\int_{S} \operatorname{pr}_{X}^{*} \omega_{X}+l \int_{F_{r}} \operatorname{pr}_{X}^{*} \omega_{X}=0
$$

As $\int_{D^{\prime}} \hat{\omega}_{r}>0$, we get that $\int_{D^{\prime}} \operatorname{pr}_{E_{\Sigma}}^{*} \omega_{\text {can }}>0$. Note that $\omega_{\text {can }}=d\left(\left(r^{2}-1\right) \alpha^{\nabla}\right)$ on $E_{\Sigma} \backslash \Sigma$, where $\alpha^{\nabla}$ is the transgression 1-form on $E_{\Sigma}$ (see Section 3). So by Stokes formula,

$$
0<\int_{D^{\prime}} \operatorname{pr}_{E_{\Sigma}}^{*} \omega_{\text {can }}=l\left(r^{2}-1\right) \int_{\gamma} \alpha=l\left(r^{2}-1\right) .
$$

This implies that $l<0$, as stated.

Step 5. Denote by $c_{1}^{T\left(E_{\Sigma}\right)} \in H^{2}\left(E_{\Sigma} ; \mathbb{Z}\right)$ the first Chern class of the tangent bundle of the symplectic manifold $\left(E_{\Sigma}, \frac{1}{k_{\mathcal{P}}} \omega_{\text {can }}\right)$. From the assumptions of the theorem we get (via the map $F_{\mathcal{P}}$ ) that

$$
c_{1}^{T\left(E_{\Sigma}\right)}=F_{\mathcal{P}}^{*} c_{1}^{M}=\frac{\lambda_{(M, \Omega)}}{k_{\mathcal{P}}}\left[\omega_{\text {can }}\right] \text { on } \pi_{2}\left(E_{\Sigma}\right) .
$$

On the other hand, from step 3 we have

$$
2 c_{1}^{T\left(E_{\Sigma} \times X\right)}([S])=\mu([D])+(-l) \mu\left(\left[F_{r}\right]\right) \leq \operatorname{dim}_{\mathbb{C}} \Sigma+\operatorname{dim}_{\mathbb{C}} X+1+2(-l) .
$$

Put $B=\left(\operatorname{pr}_{E_{\Sigma}}\right)_{*}([S]) \in \pi_{2}\left(E_{\Sigma}\right)$. Since $c_{1}^{X}$ vanishes on $\pi_{2}(X)$ we also have:

$$
c_{1}^{T\left(E_{\Sigma} \times X\right)}([S])=\frac{\lambda_{(M, \Omega)}}{k_{\mathcal{P}}} \int_{B} \omega_{\text {can }}=\frac{\lambda_{(M, \Omega)}}{k_{\mathcal{P}}}([\Sigma] \cdot B)=\frac{\lambda_{(M, \Omega)}}{k_{\mathcal{P}}}(-l) .
$$

Combining this with inequality (1) and the fact that $l<0$ we obtain

$$
\begin{aligned}
\frac{\lambda_{\left(\Sigma, \omega_{\Sigma}\right)}}{k_{\mathcal{P}}} & =\frac{\lambda_{(M, \Omega)}}{k_{\mathcal{P}}}-1 \leq \frac{\operatorname{dim}_{\mathbb{C}} \Sigma+\operatorname{dim}_{\mathbb{C}} X+1+2(-l)}{2(-l)}-1 \\
& \leq \frac{\operatorname{dim}_{\mathbb{C}} \Sigma+\operatorname{dim}_{\mathbb{C}} X+1}{2},
\end{aligned}
$$

in contradiction to the assumption of the theorem. This concludes the proof of the statement of Theorem 4.2.1 regarding simply connected Lagrangians. 
The statement regarding Lagrangians $L$ with $H_{1}(L ; \mathbb{Z})=0$. In this case the proof goes along the same lines as above. The only difference is that instead of working with the homotopy groups $\pi_{1}\left(L_{r}\right), \pi_{2}\left(E_{\Sigma} \times X, L_{r}\right)$ one uses the corresponding homology groups. For example, now we have $H_{1}\left(L_{r} ; \mathbb{Z}\right)=\mathbb{Z}[\gamma]$ rather than $\pi_{1}\left(L_{r}\right)=\mathbb{Z}[\gamma]$; the disc $D^{\prime}$ should be replaced by a surface (with boundary) representing $[D] \in H_{2}\left(E_{\Sigma} \times X, L_{r}\right)$, and so on.

We now turn to the proofs of Lemmas 5.2.1 and 5.2.2 stated in the course of the proof.

Proof of Lemma 5.2.1. We give the proof for the case of simply connected $L$. The case of $L$ satisfying $H_{1}(L ; \mathbb{Z})=0$ is very similar and even simpler.

Let $c_{1}^{L_{r}} \in H^{2}(L ; \mathbb{Z})$ be the first Chern class of the circle bundle $L_{r} \rightarrow L$. The triviality of the circle bundle $L_{r} \rightarrow L$ is equivalent to the vanishing of $c_{1}^{L_{r}}$.

Note that $H^{2}(L ; \mathbb{Z})$ has no torsion because $H_{1}(L ; \mathbb{Z})=0$. Therefore it is enough to show that $c_{1}^{L_{r}}$ vanishes in $H^{2}\left(L_{r} ; \mathbb{R}\right)$.

Denote by $i_{L}: L \rightarrow \Sigma \times X$ the inclusion. Computing in $H^{2}(L ; \mathbb{R})$ we have:

$$
c_{1}^{L_{r}}=i_{L}^{*} \operatorname{pr}_{\Sigma}^{*} c_{1}^{P_{r}}=i_{L}^{*} \operatorname{pr}_{\Sigma}^{*} c_{1}^{N_{\Sigma}}=i_{L}^{*}\left(k_{\mathcal{P}} \operatorname{pr}_{\Sigma}^{*}\left[\omega_{\Sigma}\right]\right)
$$

where $\operatorname{pr}_{\Sigma}: \Sigma \times X \rightarrow \Sigma$ is the obvious projection.

Denote by $\operatorname{pr}_{X}: \Sigma \times X \rightarrow X$ the other obvious projection. Since $\left[\omega_{X}\right]$ vanishes on $\pi_{2}(X)$ it follows that $\operatorname{pr}_{X}^{*}\left[\omega_{X}\right]$ vanishes also on $\pi_{2}(L)$. As $L$ is simply connected, the Hurewicz homomorphism $\pi_{2}(L) \rightarrow H_{2}(L ; \mathbb{Z})$ is surjective, and so $i_{L}^{*} \operatorname{pr}_{X}^{*}\left[\omega_{X}\right]$ vanishes in $H^{2}(L ; \mathbb{R})$.

Finally, from $L$ being Lagrangian for $\omega_{\Sigma} \oplus \omega_{X}$ we conclude that $i_{L}^{*} \operatorname{pr}_{\Sigma}^{*}\left[\omega_{\Sigma}\right]$ is also zero in $H^{2}(L ; \mathbb{R})$. Now we get from $(2)$ that $c_{1}^{L_{r}}=0$.

Proof of Lemma 5.2.2. Again, we present the proof for the case of $L$ being simply connected. The case $H_{1}(L ; \mathbb{Z})=0$ is completely analogous.

Note that $G_{*}: \pi_{2}\left(\left(E_{\Sigma} \backslash \Sigma\right) \times X, L_{r}\right) \rightarrow \pi_{2}\left(V \times X, G\left(L_{r}\right)\right)$ is surjective due to subcriticality. Therefore it is enough to prove that there exists $r$ such that $L_{r}$ is monotone in $\left(\left(E_{\Sigma} \backslash \Sigma\right) \times X, \hat{\omega}_{r}\right)$.

Let $A \in \pi_{2}\left(\left(E_{\Sigma} \backslash \Sigma\right) \times X, L_{r}\right)$. Denote by $\delta(A) \in \mathbb{Z}$ the unique integer such that $\partial A=\delta(A)[\gamma] \in \pi_{1}\left(L_{r}\right)$. Let $F_{r}$ be a fibre as in step 4 of the proof of Theorem 4.2.1.

With these notations the class $A-\delta(A)\left[F_{r}\right] \in \pi_{2}\left(E_{\Sigma} \times X, L_{r}\right)$ is the image of a spherical class $B \in \pi_{2}\left(E_{\Sigma} \times X\right)$ under the natural homomorphism $\pi_{2}\left(E_{\Sigma} \times X\right) \rightarrow$ $\pi_{2}\left(E_{\Sigma} \times X, L_{r}\right)$

It is easy to see that $\mu\left(\left[F_{r}\right]\right)=2$, hence $2 c_{1}^{T\left(E_{\Sigma} \times X\right)}(B)=\mu(A)-2 \delta(A)$. On the other hand, $\omega_{X}$ and $c_{1}^{X}$ vanish on $\pi_{2}(X)$ and so: 


$$
\begin{aligned}
2 c_{1}^{T\left(E_{\Sigma} \times X\right)}(B) & =2 \operatorname{pr}_{E_{\Sigma}}^{*}\left(c_{1}^{T\left(E_{\Sigma}\right)}\right)(B)=2 \lambda_{(M, \Omega)} \int_{B} \operatorname{pr}_{E_{\Sigma}}^{*}\left(\frac{1}{k_{\mathcal{P}}} \omega_{\text {can }}\right) \\
& =2 \lambda_{(M, \Omega)} \int_{B} \hat{\omega}_{r}=2 \lambda_{(M, \Omega)} \int_{A} \hat{\omega}_{r}-2 \lambda_{(M, \Omega)} \delta(A) \int_{F_{r}} \frac{1}{k_{\mathcal{P}}} \omega_{\text {can }} \\
& =2 \lambda_{(M, \Omega)} \int_{A} \hat{\omega}_{r}-2 \lambda_{(M, \Omega)} \delta(A) \frac{1}{k_{\mathcal{P}}} r^{2} .
\end{aligned}
$$

Therefore, if we choose $r=\left(\frac{k_{\mathcal{P}}}{\lambda_{(M, \Omega)}}\right)^{\frac{1}{2}}$ we have $\mu(A)=2 \lambda_{(M, \Omega)}\left[\hat{\omega}_{r}\right](A)$ for every $A \in \pi_{2}\left(\left(E_{\Sigma} \backslash \Sigma\right) \times X, L_{r}\right)$. Note that $\frac{\lambda_{(M, \Omega)}}{k_{\mathcal{P}}}=\frac{\lambda_{\left(\Sigma, \omega_{\Sigma}\right)}}{k_{\mathcal{P}}}+1>1$ and so $0<r<1$.

\subsection{Proofs of Theorems 4.2 .2 and 4.2 .3}

Proof of Theorem 4.2.3. We use the same notations as in the proof of Theorem 4.2.1. We denote by $\mathcal{P}=(M, \Omega, J ; \Sigma)$ the subcritical polarization mentioned in the statement of the theorem.

Let $L \subset\left(\Sigma \times X, \omega_{\Sigma} \oplus \omega_{X}\right)$ be a closed Lagrangian submanifold. Assume that $\pi_{1}(L)$ has no elements of infinite order. We shall prove that $H_{1}(L ; \mathbb{Z})$ contains a non-trivial cyclic subgroup whose order divides $q$.

Let $L_{r} \rightarrow L$ be the circle bundle as in the proof of Theorem 4.2.1.

Claim. The bundle $L_{r} \rightarrow L$ is not trivial.

Before we prove this, let us explain how it implies the desired statement on $H_{1}(L ; \mathbb{Z})$.

Denote by $c_{1}^{L_{r}} \in H^{2}(L ; \mathbb{Z})$ the first Chern class of the circle bundle $L_{r} \rightarrow L$ and let $i_{L}: L \rightarrow \Sigma \times X$ be the inclusion. Since $L_{r} \rightarrow L$ is not trivial, $c_{1}^{L_{r}} \neq 0$.

As $L$ is a Lagrangian submanifold, $\left.T(\Sigma \times X)\right|_{L} \cong T(L) \otimes \mathbb{C}$ and therefore $2 i_{L}^{*} c_{1}^{\Sigma \times X}=0$. Denoting by $\operatorname{pr}_{\Sigma}: \Sigma \times X \rightarrow \Sigma$ and by $\operatorname{pr}_{X}: \Sigma \times X \rightarrow X$ the obvious projections, this can be written as:

$$
2 i_{L}^{*} \operatorname{pr}_{\Sigma}^{*} c_{1}^{\Sigma}+2 i_{L}^{*} \operatorname{pr}_{X}^{*} c_{1}^{X}=0
$$

Recalling that $\nu c_{1}^{N_{\Sigma}}=\eta c_{1}^{\Sigma}, c_{1}^{L_{r}}=i_{L}^{*} \operatorname{pr}_{\Sigma}^{*} c_{1}^{N_{\Sigma}}$ and $t_{X} c_{1}^{X}=0$, we have:

$$
\begin{aligned}
q c_{1}^{L_{r}} & =i_{L}^{*} \operatorname{pr}_{\Sigma}^{*}\left(\frac{\operatorname{lcm}\left(2, \eta, t_{X}\right)}{\eta} \nu c_{1}^{N_{\Sigma}}\right) \\
& =i_{L}^{*} \operatorname{pr}_{\Sigma}^{*}\left(\operatorname{lcm}\left(2, \eta, t_{X}\right) c_{1}^{\Sigma}\right)=i_{L}^{*}\left(\operatorname{lcm}\left(2, \eta, t_{X}\right)\left(\operatorname{pr}_{\Sigma}^{*} c_{1}^{\Sigma}+\operatorname{pr}_{X}^{*} c_{1}^{X}\right)\right)=0 .
\end{aligned}
$$

Summarizing all the above we see that $c_{1}^{L_{r}} \neq 0$ but $q c_{1}^{L_{r}}=0$. This proves that $H^{2}(L ; \mathbb{Z})$ contains a non-trivial cyclic subgroup whose order divides $q$. It 
easily follows from the universal coefficient formula that the same also holds for $H_{1}(L ; \mathbb{Z})$.

It remains to prove that the circle bundle $L_{r} \rightarrow L$ is not trivial. Indeed, assume that this bundle is trivial. Note that Lemma 5.2.2 continues to hold under the assumption that all elements of $\pi_{1}(L)$ have finite order (the proof is very similar to the case $\left.\pi_{1}(L)=0\right)$. We can now apply steps 2 and 3 of the proof of Theorem 4.2.1 and in the same way obtain a disc $D \subset\left(E_{\Sigma} \backslash \Sigma\right) \times X$ such that $\hat{\omega}_{r}([D])>0$ and $\mu([D]) \leq \operatorname{dim}_{\mathbb{C}} \Sigma+\operatorname{dim}_{\mathbb{C}} X+1$.

Since the bundle $L_{r} \rightarrow L$ is trivial we have $\pi_{1}\left(L_{r}\right)=\pi_{1}(L) \times\langle[\gamma]\rangle$, where $\langle[\gamma]\rangle$ is the (infinite) cyclic group generated by $[\gamma]$. Let $l \in \mathbb{Z}$ be the unique integer such that $\partial[D]=\left(a,[\gamma]^{l}\right)$ where $a \in \pi_{1}(L)$. As in step 4 of the proof of Theorem 4.2.1 we claim that $l<0$.

To prove this recall that we are under the assumption that all the elements of $\pi_{1}(L)$ are of finite order. Let $s \in \mathbb{N}$ such that $a^{s}=1$ and choose a representative $D^{\prime}$ of $s[D] \in \pi_{2}\left(E_{\Sigma} \times X, L_{r}\right)$ such that $\partial D^{\prime}=\gamma^{s l}$. Put $S=D^{\prime} \cup_{\partial D^{\prime}}(-s l) F_{r}$. The same arguments as in Step 4 of the proof of Theorem 4.2.1 show that

$$
0<\int_{D^{\prime}} \operatorname{pr}_{E_{\Sigma}}^{*} \omega_{\text {can }}=\operatorname{sl}\left(r^{2}-1\right) \int_{\gamma} \alpha=\operatorname{sl}\left(r^{2}-1\right),
$$

hence $l<0$.

Now we proceed in an analogous way to step 5 of the proof of Theorem 4.2.1 to obtain:

$2 c_{1}^{T\left(E_{\Sigma} \times X\right)}([S])=\mu\left(\left[D^{\prime}\right]\right)+(-s l) \mu\left(\left[F_{r}\right]\right) \leq s\left(\operatorname{dim}_{\mathbb{C}} \Sigma+\operatorname{dim}_{\mathbb{C}} X+1+2(-l)\right)$, and

$c_{1}^{T\left(E_{\Sigma} \times X\right)}([S])=(-s l) \frac{\lambda_{(M, \Omega)}}{k_{\mathcal{P}}}$.

Finally, since $l<0$ we get:

$$
\begin{aligned}
\frac{\nu}{\eta}=\frac{\lambda_{\left(\Sigma, \omega_{\Sigma}\right)}}{k_{\mathcal{P}}}=\frac{\lambda_{(M, \Omega)}}{k_{\mathcal{P}}}-1 & \leq \frac{\operatorname{dim}_{\mathbb{C}} \Sigma+\operatorname{dim}_{\mathbb{C}} X+1+2(-l)}{2(-l)}-1 \\
& \leq \frac{\operatorname{dim}_{\mathbb{C}} \Sigma+\operatorname{dim}_{\mathbb{C}} X+1}{2}
\end{aligned}
$$

in contradiction to the assumption of the theorem.

This completes the proof of the claim that $L_{r} \rightarrow L$ is a non-trivial circle bundle, hence the proof of Theorem 4.2.3.

Proof of Theorem 4.2.2. The proof is analogous to the proof of Theorem 4.2.3. The required adjustments are similar to the ones needed in the proof of Theorem 4.2.1 for the case $H_{1}(L ; \mathbb{Z})=0$. One then concludes that $H_{1}(L ; \mathbb{Z})$ either contains an infinite cyclic subgroup (that is, the free part of $H_{1}(L ; \mathbb{Z})$ is non-trivial), or it has a non-trivial cyclic subgroup whose order divides $q$. By the universal coefficient formula, this is equivalent to $H^{1}(L ; \mathbb{Z} / q \mathbb{Z}) \neq 0$. 


\subsection{Proof of Theorem $\mathrm{C}$}

Proof of Theorem C. We begin with the same construction as in Steps 1 and 2 of the proof of Theorem 4.2.1, keeping the same notations. We take:

- $(M, \Omega)=\left(\mathbb{C} P^{n+1}, \sigma_{\mathrm{CP} n+1}\right)$.

- $\left(\Sigma, \omega_{\Sigma}\right) \cong\left(\mathbb{C} P^{n}, \sigma_{\mathrm{CP} n}\right)$ a linear hyperplane in $\mathbb{C} P^{n+1}$.

- $V=M \backslash \Sigma \cong \mathbb{C} P^{n+1} \backslash \mathbb{C} P^{n} \cong \operatorname{Int} B^{2 n+2}(1)$.

- $\left(X, \omega_{X}\right)=\left(\mathbb{C} P^{n}, \sigma_{\mathbb{C P}}\right)$.

Finally, put $J=J_{\mathbb{C} P^{n+1}}$ to be the standard complex structure of $\mathbb{C} P^{n+1}$. Clearly the polarization $(M, \Omega, J ; \Sigma)$ is subcritical.

Suppose that $L \subset\left(\Sigma \times X, \omega_{\Sigma} \oplus \omega_{X}\right)$ is a closed Lagrangian submanifold with $H_{1}(L ; \mathbb{Z})=0$ and $H_{2}(L ; \mathbb{Z})=0$.

Consider the circle bundle $L_{r} \rightarrow L$ as in the proof of Theorem 4.2.1. We first claim that this circle bundle is trivial. Indeed, the assumptions on $H_{1}(L ; \mathbb{Z})$ and $H_{2}(L ; \mathbb{Z})$ imply that $H^{2}(L ; \mathbb{Z})=0$, and so the first Chern class of $L_{r} \rightarrow L$ vanishes (in $H^{2}(L ; \mathbb{Z})$ ). Thus $L_{r} \rightarrow L$ is trivial.

Next, as in the proof of Theorem 4.2.1, consider the Lagrangian submanifold $G\left(L_{r}\right) \subset\left(V \times X, \Omega \oplus\left(1-r^{2}\right) \omega_{X}\right)$.

Claim. $G\left(L_{r}\right)$ is monotone. Furthermore, its minimal Maslov number defined by

$$
\mu_{\min }=\min \left\{\mu(A) \mid A \in \pi_{2}\left(V \times X, G\left(L_{r}\right)\right), \mu(A)>0\right\}
$$

satisfies $\mu_{\min }=2(n+1)$.

Before we prove this claim, let us see how this yields a contradiction. Indeed, if $\mu_{\min }=2(n+1)$ then we have $\mu_{\min }=\operatorname{dim} L_{r}+1$ and so by a theorem due to Oh [Oh-2] the Floer cohomology of $G\left(L_{r}\right)$ satisfies $H F^{i}\left(G\left(L_{r}\right) ; \mathbb{Z} / 2 \mathbb{Z}\right) \cong$ $H^{i}\left(G\left(L_{r}\right) ; \mathbb{Z} / 2 \mathbb{Z}\right)$ for every $1 \leq i \leq \operatorname{dim} L_{r}-1$. In particular:

$$
\begin{aligned}
H F^{1}\left(G\left(L_{r}\right) ; \mathbb{Z} / 2 \mathbb{Z}\right) & \cong H^{1}\left(G\left(L_{r}\right) ; \mathbb{Z} / 2 \mathbb{Z}\right) \\
& \cong H^{1}\left(L_{r} ; \mathbb{Z} / 2 \mathbb{Z}\right) \cong H^{1}\left(L \times S^{1} ; \mathbb{Z} / 2 \mathbb{Z}\right)=\mathbb{Z} / 2 \mathbb{Z} .
\end{aligned}
$$

On the other hand, since $V$ is a subcritical Stein manifold, the results of [B-C] imply that $H F^{*}\left(G\left(L_{r}\right) ; \mathbb{Z} / 2 \mathbb{Z}\right)$ completely vanishes, in contradiction to (4).

To complete the proof, it remains to show that $G\left(L_{r}\right)$ is monotone with $\mu_{\min }=$ $2(n+1)$. Let $\left[z_{0}: \ldots: z_{n+1}\right]$ be homogeneous coordinates on $M=\mathbb{C} P^{n+1}$ and write $\Sigma$ as the hyperplane $\left\{z_{0}=0\right\}$. With this choice of $\Sigma$ the skeleton of the polarization $\mathcal{P}=\left(\mathbb{C} P^{n+1}, \sigma_{\mathrm{CP} n+1}, J ; \Sigma\right)$ is just $\Delta=\{[1: 0: \ldots: 0]\}$ (see Example 2.2.1).

The circle bundle $P_{r} \rightarrow \Sigma$ can be naturally identified with the sphere

$$
P_{r}=\left\{\left.\left(z_{1}, \ldots, z_{n+1}\right) \in \mathbb{C}^{n+1}\left|\sum_{j=1}^{n+1}\right| z_{j}\right|^{2}=1-r^{2}\right\} \cong S^{2 n+1},
$$


where the projection $P_{r} \rightarrow \Sigma$ is given by $P_{r} \ni\left(z_{1}, \ldots, z_{n+1}\right) \mapsto\left[0: z_{1}: \ldots\right.$ : $\left.z_{n+1}\right] \in \Sigma$. With these identifications the restriction of the map $F_{\mathcal{P}}$ to $P_{r}$ is just:

$$
F_{\mathcal{P}}\left(z_{1}, \ldots, z_{n+1}\right)=\left[r: z_{1}: \ldots: z_{n+1}\right] \text { for every }\left(z_{1}, \ldots, z_{n+1}\right) \in P_{r}
$$

Pick a point $\left(p_{1}, p_{2}\right) \in L$ and write $p_{1}=\left[0: z_{1}: \ldots: z_{n+1}\right]$, where $\left(z_{1}, \ldots, z_{n+1}\right)$ are normalized to be in $P_{r}$, that is $\sum_{j=1}^{n+1}\left|z_{j}\right|^{2}=1-r^{2}$. Let $S \subset M \cong \mathbb{C} P^{n+1}$ be the projective line which connects the point $p_{1}$ and the point $[1: 0: \ldots: 0]$ forming the skeleton $\Delta$, namely

$$
S=\left\{\left[\lambda_{0}: \lambda_{1} z_{1}: \ldots: \lambda_{1} z_{n+1}\right] \mid\left[\lambda_{0}: \lambda_{1}\right] \in \mathbb{C} P^{1}\right\}
$$

Consider now the decomposition $S=D_{+}^{\prime} \cup D_{-}^{\prime}$ into two discs, where:

$$
\begin{aligned}
& D_{+}^{\prime}=\left\{\left[\lambda_{0}: \lambda_{1} z_{1}: \ldots: \lambda_{1} z_{n+1}\right]|| \lambda_{0}|\leq r| \lambda_{1} \mid\right\}, \\
& D_{-}^{\prime}=\left\{\left[\lambda_{0}: \lambda_{1} z_{1}: \ldots: \lambda_{1} z_{n+1}\right]|| \lambda_{0}|\geq r| \lambda_{1} \mid\right\} .
\end{aligned}
$$

Finally, put $D_{+}=D_{+}^{\prime} \times p_{2}$ and $D_{-}=D_{-}^{\prime} \times p_{2}$. Note that both discs $D_{+}, D_{-}$ have their boundaries on the Lagrangian submanifold $G\left(L_{r}\right)$. Moreover, in the notations of the proof of Theorem 4.2.1, the disc $D_{+}$is precisely the image $G\left(F_{r}\right)$ of the fibre $F_{r}$ of the disc bundle $\overline{E_{\Sigma}(r)} \times X \rightarrow \Sigma \times X$, and its (oriented) boundary $\partial D_{+}$is just $G(\gamma)$. Similarly, the disc $D_{-}$lies in $V \times X$, and its boundary is a fibre of the circle bundle $G\left(L_{r}\right) \rightarrow L$ but with the opposite orientation, namely $G\left(\gamma^{-1}\right)$.

Note that

$$
\pi_{2}\left(V \times \mathbb{C} P^{n}, G\left(L_{r}\right)\right) \cong \mathbb{Z} A \oplus \mathbb{Z}\left[D_{-}\right]
$$

where $A$ is the image of the class $[\mathrm{pt} \times$ line $] \in \pi_{2}\left(V \times \mathbb{C} P^{n}\right)$ under the natural homomorphism $\pi_{2}\left(V \times \mathbb{C} P^{n}\right) \rightarrow \pi_{2}\left(V \times \mathbb{C} P^{n}, L_{r}\right)$. We have $\mu\left(D_{+}\right)=2$, hence

$$
\mu\left(D_{-}\right)=2 c_{1}^{M}(S)-\mu\left(D_{+}\right)=2(n+1) .
$$

Also, $\mu(A)=2 c_{1}^{\mathbb{C} P^{n}}(A)=2(n+1)$. Since the symplectic form $\Omega \oplus\left(1-r^{2}\right) \omega_{X}$ takes the value $1-r^{2}$ on both $A$ and $D_{-}$, it follows that $G\left(L_{r}\right) \subset V \times \mathbb{C} P^{n}$ is monotone with $\mu_{\min }=2(n+1)$. 


\section{Gromov radius}

In this section we prove Theorems $\mathrm{F}$ and $\mathrm{G}$ from the introduction.

\subsection{Isotopies of subcritical $\mathbf{C W}$-complexes and proof of Theorem $\mathbf{F}$}

An important ingredient in the proof of Theorem $\mathrm{F}$ is the following isotopy theorem, essentially due to Laudenbach [Lau].

Theorem 6.1.1 (Isotopy Theorem). Let $(M, \Omega)$ be a symplectic manifold (without boundary, but possibly non-compact) and $\Delta \subset(M, \Omega)$ an embedded finite $C W$ complex with $\operatorname{dim} \Delta<\frac{1}{2} \operatorname{dim} M$. Let $B \subset M$ be a closed subset. Suppose that there exists a (continuous) homotopy $\psi_{t}: \Delta \rightarrow M$ such that $\psi_{0}$ is the inclusion and $\psi_{1}(\Delta) \cap B=\emptyset$. Then there exists a compactly supported Hamiltonian isotopy $\Psi_{t}:(M, \Omega) \rightarrow(M, \Omega)$ with $\Psi_{0}=\mathbb{1}$ and $\Psi_{1}(\Delta) \cap B=\emptyset$.

This theorem is a slight modification of Theorem IV from [Lau] (see also Theorem I there) and can be proved in almost the same way. Let us also remark that the above theorem and its proof belong to the framework of Gromov's h-principle (see $[\mathrm{Gr}-1])$.

Now let $\mathcal{P}=(M, \Omega, J ; \Sigma)$ be a subcritical polarization. By Corollary 3.4 there exists a subcritical isotropic CW-complex $\Delta \subset M \backslash \Sigma$ such that $(M \backslash \Delta, \Omega)$ is symplectomorphic to the standard symplectic disc bundle $\left(E_{\Sigma}, \frac{1}{k_{\mathcal{P}}} \omega_{\text {can }}\right)$.

The following result is an immediate consequence of the Isotopy Theorem and Corollary 3.4. Here by a smoothly contractible subset of $M$ we mean a subset which can be isotoped (via an ambient smooth isotopy) into an arbitrarily small coordinate neighbourhood in $M$. For example, a disjoint union of embedded closed balls is always smoothly contractible.

Proposition 6.1.2. Let $\mathcal{P}=(M, \Omega, J ; \Sigma)$ be a subcritical polarization of degree $k_{\mathcal{P}}$. Let $(N, \nu)$ be a symplectic manifold, possibly non-compact or with boundary. Suppose that there exists a symplectic embedding $f:(N, \nu) \rightarrow(M, \Omega)$ such that $f(N)$ is smoothly contractible in $M$. Then there exists a symplectic embedding $(N, \nu) \rightarrow\left(E_{\Sigma}, \frac{1}{k_{\mathcal{P}}} \omega_{\text {can }}\right)$.

In particular, $\rho_{G}(M, \Omega)=\rho_{G}\left(E_{\Sigma}, \frac{1}{k_{\mathcal{P}}} \omega_{\text {can }}\right)$.

Remark. Note that the inequality $\rho_{G}\left(E_{\Sigma}, \frac{1}{k_{\mathcal{P}}} \omega_{\text {can }}\right) \leq \rho_{G}(M, \Omega)$ holds for any polarization, subcritical or not. However, in the critical case this inequality may be strict. This happens e.g. for the degree 2 polarization $\left(\mathbb{C} P^{n}, \sigma, J_{\mathbb{C} P^{n}} ; \Sigma\right)$ where $\Sigma$ is a smooth quadric (see $[\mathrm{Bi}-1]$ ). The reason is that in this case $\Delta$ contains Lagrangian cells, for which Theorem 6.1.1, and Gromov's h-principle in general, 
do not apply. This discrepancy between the two Gromov radii and its implications have been studied in [Bi-1].

Proof of Theorem $F$. Let $\mathcal{P}=(M, \Omega, J ; \Sigma)$ be the subcritical polarization of degree $k_{\mathcal{P}}$ as in the statement of the theorem.

In view of Proposition 6.1 .2 we have $\rho_{G}(M, \Omega)=\rho_{G}\left(E_{\Sigma}, \frac{1}{k_{\mathcal{P}}} \omega_{\text {can }}\right)$. On the other hand, it is proved in [Bi-1] (Proposition 5.A, see also Theorem 4.A and its proof) that under the conditions of the theorem we have $\rho_{G}\left(E_{\Sigma}, \omega_{\text {can }}\right) \leq 1$, hence $\rho_{G}\left(E_{\Sigma}, \frac{1}{k_{\mathcal{P}}} \omega_{\text {can }}\right) \leq \frac{1}{k_{\mathcal{P}}}$

The converse estimate when $\mathcal{O}_{\Sigma}(\Sigma) \rightarrow \Sigma$ is base point free follows from the results of $[\mathrm{Bi}-1]$ (see Lemma $5 . \mathrm{B}$ there).

\subsection{Seshadri constants and proof of Theorem G}

Before we go to the proof we need a short tour into the theory of Seshadri constants. Seshadri constants were defined by Demailly [Dem]. We refer the reader also to $[\mathrm{E}-\mathrm{L}],[\mathrm{E}-\mathrm{K}-\mathrm{L}]$ and the references therein for further details and interesting results concerning these constants.

Let $M$ be a complex manifold, and $\mathcal{L} \rightarrow M$ an ample line bundle. The Seshadri constant of $\mathcal{L}$ at the point $p \in M$ is the following non-negative real number:

$$
\mathcal{E}(\mathcal{L}, p)=\inf _{C \ni p} \frac{\int_{C} c_{1}^{\mathcal{L}}}{\operatorname{mult}_{p} C},
$$

where the infimum is taken over all irreducible holomorphic curves $C$ passing through the point $p$. Since this quantity may depend on the point $p$, it is useful to define the following more global invariant:

$$
\mathcal{E}(\mathcal{L})=\sup _{p \in M} \mathcal{E}(\mathcal{L}, p)
$$

which will be called the global Seshadri constant of $\mathcal{L}$.

Given an ample line bundle $\mathcal{L} \rightarrow M$ over a complex manifold $(M, J)$, the cohomology class $c_{1}^{\mathcal{L}}$ can be represented by a $J$-compatible Kähler form $\Omega_{\mathcal{L}}$ by taking the curvature of $\mathcal{L}$ with respect to a suitable metric connection. Note that the symplectomorphism type of $\left(M, \Omega_{\mathcal{L}}\right)$ depends only on the cohomology class $c_{1}^{\mathcal{L}}$. This follows easily by Moser's argument, since the space of $J$-compatible symplectic forms on $M$ is linearly convex.

The next proposition establishes a relation between Seshadri constants and the Gromov radius (cf. [Laz] and also [Bi-3]):

Proposition 6.2.1. Let $\mathcal{L} \rightarrow M$ be an ample line bundle over a complex manifold $(M, J)$. Then

$$
\rho_{G}\left(M, \Omega_{\mathcal{L}}\right) \geq \mathcal{E}(\mathcal{L})
$$


Proof. Let $\pi: \widetilde{M}_{p} \rightarrow M$ be the complex blow-up of $M$ at a point $p \in M$, with exceptional divisor $E$ over $p$. Then it is not hard to see that (see [Dem]):

$$
\mathcal{E}(\mathcal{L}, p)=\sup \left\{t \in \mathbb{R} \mid \text { The } \mathbb{R} \text {-divisor } \pi^{*} \mathcal{L}-t E \text { is nef }\right\} .
$$

Recall that an $\mathbb{R}$-divisor $D$ on an algebraic variety is called nef (numerically effective) if it lies in the closure of the ( $\mathbb{R}$-)ample cone.

Now let $e \in H^{2}\left(\widetilde{M}_{p}\right)$ be the Poincaré dual to $E$. Clearly (5) is equivalent to:

$\mathcal{E}(\mathcal{L}, p)=\sup \left\{t \in \mathbb{R} \mid\right.$ The cohomology class $\pi^{*}\left[\Omega_{\mathcal{L}}\right]-t e \in H^{1,1}\left(\widetilde{M}_{p}\right)$ is Kähler $\}$.

Performing symplectic blowing-down (see e.g. [M-P], Corollary 2.1.D) we conclude that $\rho_{G}\left(M, \Omega_{\mathcal{L}}\right) \geq \mathcal{E}(\mathcal{L}, p)$. Since this is true for every $p \in M$, the proposition follows.

We now turn to the

Proof of Theorem $G$. Since $[\Omega] \in H^{2}(M ; \mathbb{Z}) \cap H^{1,1}(M, J)$, there exists a holomorphic line bundle $\mathcal{L} \rightarrow(M, J)$ with $c_{1}^{\mathcal{L}}=[\Omega]$.

By the results of $[\mathrm{E}-\mathrm{K}-\mathrm{L}]$, for a "very general" point $p \in M$ we have the following uniform bound:

$$
\mathcal{E}(\mathcal{L}, p) \geq \frac{1}{\operatorname{dim}_{\mathbb{C}} M} .
$$

Combining this with Proposition 6.2.1 we get:

$$
\rho_{G}(M, \Omega) \geq \mathcal{E}(\mathcal{L}) \geq \mathcal{E}(\mathcal{L}, p) \geq \frac{1}{\operatorname{dim}_{\mathbb{C}} M} .
$$

On the other hand by Theorem F, $\rho_{G}(M, \Omega) \leq \frac{1}{k_{\mathcal{P}}}$. Therefore $k_{\mathcal{P}} \leq \operatorname{dim}_{\mathbb{C}} M$. In particular, $k_{\mathrm{hol}}(M, J,[\Omega]) \leq \operatorname{dim}_{\mathbb{C}} M$.

\section{Proof of the Desingularization Theorem}

Idea of the proof. We will show that $\phi=-\log \|s\|^{2}$ has two types of critical points. The first type are $\epsilon$-small perturbations of critical points of $-\log \left\|s_{1} \otimes s_{2}\right\|^{2}$ outside $\Sigma_{1} \cup \Sigma_{2}$, which have index $<n=\operatorname{dim}_{\mathbb{C}} M$ by assumption 1 . The second type of critical points occur near $\Sigma_{1} \cap \Sigma_{2}$. Up to small perturbations, they can be modeled as critical points of functions

$$
\left(z_{1}, \ldots, z_{n}\right) \mapsto-\log \left|z_{1} z_{2}+\epsilon\right|^{2}-\log \left\|s_{0}\left(z_{3}, \ldots, z_{n}\right)\right\|^{2}
$$

on a neighbourhood of 0 in $\mathbb{C}^{n}$ such that $\Sigma_{1} \cap \Sigma_{2}$ is described by $\left\{z_{1}=z_{2}=0\right\}$. The first term has a critical point of index 2 , whereas the second term has critical points of index $<n-2$ by assumption 2 , so the second type of critical points also have index $<n$. Let us now make these arguments precise. 
Proof of Theorem 2.3.1. Step 1. We cover $M$ by finitely many holomorphic charts on which $\mathcal{L}_{1}, \mathcal{L}_{2}$ are trivial. This allows us to view $s_{1}, s_{2}, s_{1} \otimes s_{2}=s_{1} s_{2}$ as functions and speak of $\partial s_{i}$ etc. In view of the transversality assumptions, there exist arbitrarily small constants $0<\delta<\rho$ with the following properties:

- For $i=1,2, V_{i}=\left\{\left|s_{i}\right|<\delta\right\}$ is a neighbourhood of $\Sigma_{i}$ on which $\left|\partial s_{i}\right| \geq \rho$.

- $V=\left\{\left|s_{1}\right|^{2}+\left|s_{2}\right|^{2}<\rho^{2}\right\}$ is a neighbourhood of $D$ on which

$$
\left|a_{1} \partial s_{1}+a_{2} \partial s_{2}\right|^{2} \geq \rho\left(\left|a_{1}\right|^{2}+\left|a_{2}\right|^{2}\right) \quad \text { for all } a_{1}, a_{2} \in \mathbb{C} \text {. }
$$

- $U=\left\{\left|s_{1}\right|^{2}+\left|s_{2}\right|^{2}<\rho^{2},\left|s_{0}\right|<\rho\right\}$ is a neighbourhood of $Z$ on which

$$
\left|a_{0} \partial s_{0}+a_{1} \partial s_{1}+a_{2} \partial s_{2}\right|^{2} \geq \rho\left(\left|a_{0}\right|^{2}+\left|a_{1}\right|^{2}+\left|a_{2}\right|^{2}\right) \quad \text { for all } a_{1}, a_{2}, a_{3} \in \mathbb{C} \text {. }
$$

These properties imply the following:

- On $M \backslash\left(V_{1} \cup V_{2}\right):\left|s_{1} s_{2}\right| \geq \delta^{2}$.

- On $V_{1} \backslash V$ : From $\rho^{2} \leq\left|s_{1}\right|^{2}+\left|s_{2}\right|^{2}<\delta^{2}+\left|s_{2}\right|^{2}$ we infer

$$
\begin{aligned}
\left|\partial\left(s_{1} s_{2}\right)\right| & =\left|s_{1} \partial s_{2}+s_{2} \partial s_{1}\right| \geq\left|s_{2}\right|\left|\partial s_{1}\right|-\left|s_{1}\right|\left|\partial s_{2}\right| \\
& \geq \sqrt{\rho^{2}-\delta^{2}} \rho-c \delta \geq \frac{\rho^{2}}{2}
\end{aligned}
$$

for $\delta$ sufficiently small, and similarly on $V_{2} \backslash V$. Here and in the following we denote by $c$ a generic constant independent of $\epsilon, \delta, \rho$.

Step 2. For $\epsilon>0$ small, $s$ is transverse to the zero section.

Proof. On $M \backslash\left(V_{1} \cup V_{2}\right):|s| \geq \delta^{2}-\epsilon\left|s_{0}\right|>0$ for $\epsilon$ small.

On $V_{i} \backslash V$ : By step $1,|\partial s| \geq \frac{\rho^{2}}{2}-\epsilon\left|\partial s_{0}\right| \geq \frac{\rho^{2}}{4}>0$ for $\epsilon$ small.

On $V \backslash U$ : If $s=0$ then $\left|s_{1} s_{2}\right| \geq \epsilon \rho$ and hence

$$
\begin{aligned}
|\partial s| & \geq\left|s_{1} \partial s_{2}+s_{2} \partial s_{1}\right|-\epsilon\left|\partial s_{0}\right| \geq \rho^{1 / 2} \sqrt{\left|s_{1}\right|^{2}+\left|s_{2}\right|^{2}}-c \epsilon \\
& \geq \rho^{1 / 2} \sqrt{\left|s_{1} s_{2}\right|}-c \epsilon \geq \rho \epsilon^{1 / 2}-c \epsilon \\
& >0 \quad \text { for } \epsilon \text { small. }
\end{aligned}
$$

On $U:|\partial s|^{2} \geq \rho\left(\left|s_{1}\right|^{2}+\left|s_{2}\right|^{2}+\epsilon^{2}\right)>0$.

Step 3. The critical points of $\phi=-\log \|s\|_{\mathcal{L}}^{2}$ lie in $M \backslash\left(V_{1} \cup V_{2}\right)$ or in $V \backslash U$. The critical points in $M \backslash\left(V_{1} \cup V_{2}\right)$ have index $<n$. The critical points in $V \backslash U$ satisfy $\rho\left(\left|s_{1}\right|^{2}+\left|s_{2}\right|^{2}\right) \leq c \epsilon^{2}$.

Proof. In holomorphic charts write the metric on $\mathcal{L}$ as \|\|$_{\mathcal{L}}^{2}=e^{h}||$ where || is the Euclidean metric and $h$ a real function. Then $\phi=-\log |s|^{2}-h$ and $\partial \phi=$ $-\frac{1}{s} \partial s-\partial h$. So at a critical point, $\partial s=-s \partial h$.

On $V_{i} \backslash V$ : At a critical point, by step 2 ,

$$
\frac{\rho^{2}}{4} \leq|\partial s|=|\partial h||s| \leq c\left(\delta+\epsilon\left|s_{0}\right|\right),
$$


which is impossible for $\delta, \epsilon$ small.

On $U$ : At a critical point,

$$
\begin{aligned}
\rho\left(\left|s_{1}\right|^{2}+\left|s_{2}\right|^{2}+\epsilon^{2}\right) & \leq|\partial s|^{2} \leq|\partial h|^{2}\left(\left|s_{1} s_{2}\right|+\epsilon\left|s_{0}\right|\right)^{2} \\
& \leq c\left(\left|s_{1} s_{2}\right|^{2}+\epsilon^{2} \rho^{2}\right) \leq c \rho^{2}\left|s_{1}\right|^{2}+c \rho^{2} \epsilon^{2},
\end{aligned}
$$

which is impossible for $c \rho<1$.

On $M \backslash\left(V_{1} \cup V_{2}\right)$ : The critical points of $-\log \left\|s_{1} s_{2}\right\|_{\mathcal{L}}^{2}$ are non-degenerate of index $<n$, so the same is true for the critical points of $-\log \|s\|_{\mathcal{L}}^{2}$ for $\epsilon$ small.

On $V \backslash U$ : At a critical point, by step 2,

$$
\begin{aligned}
\rho^{1 / 2} \sqrt{\left|s_{1}\right|^{2}+\left|s_{2}\right|^{2}}-c \epsilon & \leq|\partial s| \leq|\partial h|\left(\left|s_{1} s_{2}\right|+\epsilon\left|s_{0}\right|\right) \\
& \leq c\left(\left|s_{1}\right|^{2}+\left|s_{2}\right|^{2}+\epsilon\right) \leq c \rho^{1 / 2} \sqrt{\left|s_{1}\right|^{2}+\left|s_{2}\right|^{2}}+c \epsilon,
\end{aligned}
$$

which implies $\rho^{1 / 2} \sqrt{\left|s_{1}\right|^{2}+\left|s_{2}\right|^{2}} \leq c \epsilon$ for $\rho$ sufficiently small.

Step 4. The critical points of $\phi$ in $V \backslash U$ are in one-to-one correspondence with the critical points of $\phi_{0}=-\log \left\|\left.s_{0}\right|_{D}\right\|_{\mathcal{L}}^{2}: D \rightarrow \mathbb{R}$ and have index $<n$.

Proof. For $\rho$ sufficiently small we can cover $V$ by holomorphic charts $\left\{\left(z_{1}, \ldots, z_{n}\right) \in\right.$ $\left.\left.\mathbb{C}^{n}|| z_{1}\right|^{2}+\left|z_{2}\right|^{2}<\rho^{2},\left|z_{3}\right|^{2}+\cdots+\left|z_{n}\right|^{2}<\rho^{2}\right\}$ in which $s_{1}(z)=z_{1}, s_{2}(z)=z_{2}$. Then

$$
\begin{gathered}
\frac{\partial \phi}{\partial z_{1}}=-\frac{1}{s}\left(z_{2}+\epsilon \frac{\partial s_{0}}{\partial z_{1}}\right)-\frac{\partial h}{\partial z_{1}}, \\
\frac{\partial \phi}{\partial z_{2}}=-\frac{1}{s}\left(z_{1}+\epsilon \frac{\partial s_{0}}{\partial z_{2}}\right)-\frac{\partial h}{\partial z_{2}}, \\
\frac{\partial \phi}{\partial z_{j}}=-\frac{1}{s} \epsilon \frac{\partial s_{0}}{\partial z_{j}}-\frac{\partial h}{\partial z_{j}} \quad \text { for } j=3, \ldots, n, \\
\frac{\partial^{2} \phi}{\partial z_{1} \partial z_{2}}=-\frac{1}{s}\left(1+\epsilon \frac{\partial^{2} s_{0}}{\partial z_{1} \partial z_{2}}\right)+\frac{1}{s^{2}}\left(z_{2}+\epsilon \frac{\partial s_{0}}{\partial z_{1}}\right)\left(z_{1}+\epsilon \frac{\partial s_{0}}{\partial z_{2}}\right)-\frac{\partial^{2} h}{\partial z_{1} \partial z_{2}} \quad \text { etc. }
\end{gathered}
$$

By step 3, $\rho\left(\left|s_{1}\right|^{2}+\left|s_{2}\right|^{2}\right) \leq c \epsilon^{2}$ at a critical point, so $s=\epsilon s_{0}+O\left(\epsilon^{2}\right)$. It follows that the expansion of the second order derivatives at a critical point in orders of $\epsilon$ looks as follows:

$$
\frac{\partial^{2} \phi}{\partial z_{1} \partial z_{2}}=-\frac{1}{\epsilon s_{0}}+O(1), \quad \frac{\partial^{2} \phi}{\partial \bar{z}_{1} \partial \bar{z}_{2}}=-\frac{1}{\epsilon \bar{s}_{0}}+O(1)
$$

and all other second order derivatives are of $O(1)$, i.e. zero order in $\epsilon$. Thus the Hessian at a critical point $z$ has the form

$$
\operatorname{Hess}_{z} \phi=\left(\begin{array}{ccc}
\frac{1}{\epsilon} A+\tilde{A} & B \\
B^{t} & C
\end{array}\right)+O(\epsilon)
$$


where the matrices $A, \tilde{A}, B, C$ are of $O(1)$. Moreover, the real $4 \times 4$-matrix $A$ corresponds to the quadratic form

$$
\begin{aligned}
\left(w_{1}, w_{2}\right) & \mapsto-\frac{1}{s_{0}} w_{1} w_{2}-\frac{1}{\bar{s}_{0}} \bar{w}_{1} \bar{w}_{2} \\
& =2 \operatorname{Im}\left(\frac{w_{1}}{s_{0}}\right) \operatorname{Im}\left(w_{2}\right)-2 \operatorname{Re}\left(\frac{w_{1}}{s_{0}}\right) \operatorname{Re}\left(w_{2}\right),
\end{aligned}
$$

which is non-degenerate of index 2 .

The second order partial derivatives for $3 \leq j, k \leq n$ are given by

$$
\frac{\partial^{2} \phi}{\partial z_{j} \partial z_{k}}=\frac{\partial^{2}}{\partial z_{j} \partial z_{k}}\left(-\log \left|s_{0}\right|^{2}-h\right)+O(\epsilon)
$$

and similarly for $\frac{\partial^{2} \phi}{\partial \bar{z}_{j} \partial z_{k}}$ and $\frac{\partial^{2} \phi}{\partial \bar{z}_{j} \partial \bar{z}_{k}}$. So the matrix $C$ is given by

$$
C=\operatorname{Hess}_{\left(z_{3}, \ldots, z_{n}\right)} \phi_{0},
$$

which is non-degenerate of index $<n-2$. This proves that all critical points of $\phi$ in $V \backslash U$ are non-degenerate of index $<n$.

It remains to show the one-to-one correspondence between critical points of $\phi$ and $\phi_{0}$. To see this, consider for fixed $\left(z_{3}, \ldots, z_{n}\right)$ the map

$$
f_{\epsilon}\left(z_{1}, z_{2}\right)=\epsilon\left(\frac{\partial \phi}{\partial z_{1}}(z), \frac{\partial \phi}{\partial z_{2}}(z)\right)=\frac{1}{s_{0}}\left(z_{2}, z_{1}\right)+O(\epsilon)
$$

on the domain $N=\left\{\left|z_{1}\right|^{2}+\left|z_{2}\right|^{2} \leq \rho^{2}\right\}$. For $\epsilon$ small there are no solutions of $f_{\epsilon}=0$ on the boundary $\partial N$. So the mapping degree of $f_{\epsilon}$ equals the degree of $f_{0}\left(z_{1}, z_{2}\right)=\frac{1}{s_{0}}\left(z_{2}, z_{1}\right)$ which equals 1 . Since the matrix $A$ is non-degenerate of index 2 , all zeroes of $f_{\epsilon}$ are non-degenerate of local degree $(-1)^{2}=1$. So $f_{\epsilon}$ has a unique zero in $N$. The non-degeneracy of the matrix $C$ implies that near every critical point of $\phi_{0}$ there exists a unique point $\left(z_{3}, \ldots, z_{n}\right)$ at which $\frac{\partial \phi}{\partial z_{3}}=\cdots=\frac{\partial \phi}{\partial z_{n}}=0$.

\section{Discussion}

Symplectic packings. In analogy to the Gromov radius, McDuff and Polterovich defined and studied in $[\mathrm{M}-\mathrm{P}]$ (see also [Bi-2]) the quantity

$$
v_{N}(M, \Omega)=\sup _{r} \frac{\operatorname{Vol}\left(\operatorname{Image} \varphi_{r}\right)}{\operatorname{Vol}(M, \Omega)},
$$

where the supremum is taken over all radii $r$ for which there exists a symplectic embedding $\varphi_{r}: B^{2 n}(r) \amalg \ldots \amalg B^{2 n}(r) \rightarrow(M, \Omega)$ of a disjoint union of $N$ balls of radius $r$.

The following is an immediate consequence of Proposition 6.1.2. 
Proposition 8.1. Let $\mathcal{P}=(M, \Omega, J ; \Sigma)$ be a subcritical polarization of degree $k_{\mathcal{P}}$. If a disjoint union of closed balls $B^{2 n}\left(r_{1}\right) \coprod \cdots \coprod B^{2 n}\left(r_{N}\right) \rightarrow(M, \Omega)$ embeds symplectically into $(M, \Omega)$, then it also embeds symplectically into the standard symplectic disc bundle $\left(E_{\Sigma}, \frac{1}{k_{\mathcal{P}}} \omega_{\text {can }}\right)$. In particular, $v_{N}(M, \Omega)=v_{N}\left(E_{\Sigma}, \frac{1}{k_{\mathcal{P}}} \omega_{\text {can }}\right)$ for every $N \geq 1$.

This result reduces the packing problem from $(M, \Omega)$ to a standard symplectic disk bundle, where it should be more tractable. In dimension 4 the numbers $v_{N}$ are known for many cases (see $[\mathrm{M}-\mathrm{P}],[\mathrm{Bi}-2]$ ). In dimension $>4$, even on disk bundles the packing problem seems out of reach with the methods currently available.

Spaces of symplectic embeddings. Given a vector of positive numbers $\underline{r}=\left(r_{1}, \ldots, r_{N}\right)$ denote by $\operatorname{Emb}(M, \Omega ; \underline{r})$ the space of (unparametrised) symplectic embeddings of the disjoint union $B^{2 n}\left(r_{1}\right) \coprod \cdots \coprod B^{2 n}\left(r_{N}\right)$ into $(M, \Omega)$, equipped with the $C^{\infty}$ topology. A natural question, going back to the beginning of symplectic topology, is whether or not these spaces are connected. At present this problem is widely open in general (see [McD-1, McD-2, La, Bi-4, McD-3] for partial results in dimension 4).

Let us denote by $i: \operatorname{Emb}\left(E_{\Sigma}, \frac{1}{k_{\mathcal{P}}} \omega_{\text {can }} ; \underline{r}\right) \hookrightarrow \operatorname{Emb}(M, \Omega ; \underline{r})$ the natural inclusion coming from the embedding described in Corollary 3.4. Proposition 6.1.2 states that for symplectic manifolds $(M, \Omega)$ that admit a subcritical polarization the induced map

$$
i_{\#}: \pi_{0}\left(E m b\left(E_{\Sigma}, \frac{1}{k_{\mathcal{P}}} \omega_{\mathrm{can}} ; \underline{r}\right)\right) \rightarrow \pi_{0}(\operatorname{Emb}(M, \Omega ; \underline{r}))
$$

is surjective. In fact, a 1-parametric version of Theorem 6.1.1 shows that this map is also injective, hence an isomorphism.

For higher homotopy groups, one expects the induced map on $\pi_{k}$ to be an isomorphism provided that $k \leq 2 n-\operatorname{dim}\left(\Delta_{\mathcal{P}}\right)-2$, where $\Delta_{\mathcal{P}}$ is the skeleton of the polarization $\mathcal{P}$.

Symplectic capacities. Symplectic capacities have played an important role in the development of symplectic geometry. One example is the Hofer-Zehnder capacity $c_{H Z}$ (see [H-Z] for the definition) which is closely linked to Hamiltonian dynamics. For instance, its finiteness implies the Weinstein conjecture for convex hypersurfaces. However, the Hofer-Zehnder capacity has been computed only in very few cases.

A variation of this is the capacity $c_{H Z}^{0} \leq c_{H Z}$, where in its definition the supremum is only taken over Hamiltonians $H$ for which the set $\{H<\max H\}$ is smoothly contractible in $M$. For the latter capacity, the Isotopy Theorem yields the following result: 
Proposition 8.2. For a subcritical polarization $\mathcal{P}=(M, \Omega, J ; \Sigma)$,

$$
c_{H Z}^{0}(M, \Omega)=c_{H Z}^{0}\left(E_{\Sigma}, \frac{1}{k_{\mathcal{P}}} \omega_{\text {can }}\right) .
$$

This reduces the computation of $c_{H Z}^{0}$ from $(M, \Omega)$ to the standard disk bundle, where it may be approached by the methods of $[\mathrm{H}-\mathrm{V}]$.

Degree of subcritical polarizations. All our examples of subcritical polarizations have degree $k_{\mathcal{P}}=1$. We conjecture that this is true for every subcritical polarization. Note that this conjecture would improve the bound of Theorem $\mathrm{G}$ from $k_{\text {hol }}(M, J,[\Omega]) \leq \operatorname{dim}_{\mathbb{C}} M$ to $k_{\text {hol }}(M, J,[\Omega])=1$.

There appear to be two ways to prove this conjecture. One using symplectic homology (see e.g. [C-F-H]), the other using contact homology (see [El-3]). While the first approach seems simpler, the second one has the advantage of giving more information than $k_{\mathcal{P}}=1$.

Holomorphic spheres in subcritical polarizations. All of our examples of subcritical polarizations are uniruled in the sense that through every point there passes a nonconstant holomorphic sphere. We conjecture that this is true for every subcritical polarization. More precisely, we expect that on every subcritical polarization some spherical Gromov-Witten invariant of a point is nonzero. This conjecture may also be approached via contact homology ([El-3]).

\section{References}

[A-L-P] M. Audin, F. Lalonde and L. Polterovich, Symplectic rigidity: Lagrangian submanifolds. In Holomorphic curves in symplectic geometry. Edited by M. Audin and J. Lafontaine, Progress in Mathematics, 117, Birkhäuser Verlag, Basel, 1994.

[B-P-V] W. Barth, C. Peters and A. Van de Ven, Compact Complex Surfaces, Springer-Verlag, 1984.

[Bi-1] P. Biran, Lagrangian barriers and symplectic embeddings, Geom. Funct. Anal. 11 (2001), no. 3, 407-464.

[Bi-2] P. Biran, A stability property of symplectic packing, Invent. Math. 136 (1999), 123155 .

[Bi-3] P. Biran, Constructing new ample divisors out of old ones, Duke Math. J. 98 (1999), 113-135.

[Bi-4] P. Biran, Connectedness of spaces of symplectic embeddings, Internat. Math. Res. Notices no. 10 (1996), 487-491.

[B-C] P. Biran and K. Cieliebak, Lagrangian embeddings into subcritical Stein manifolds, to appear in Israel J. Math.

[C-F-H] K. Cieliebak, A. Floer and H. Hofer, Symplectic homology II: A general construction Math. Zeitschrift 218 (1995), 103-122.

[Dem] J.-P. Demailly, $L^{2}$-vanishing theorems for positive line bundles and adjunction theory, In: Transcendental methods in Algebraic Geometry, F. Catanese and C. Ciliberto eds., Lect. Notes in Math. 1646, pp. 1-97. Springer Verlag, 1996. 
[El-1] Y. Eliashberg, Topological characterization of Stein manifolds of dimension $>2$, Internat. J. Math. 1 (1990), no. 1, 29-46.

[El-2] Y. Eliashberg, Symplectic geometry of plurisubharmonic functions, In Gauge theory and symplectic geometry (Montreal, PQ, 1995), 49-67, Kluwer Acad. Publ., Dordrecht, 1997.

[El-3] Y. Eliashberg, Invariants in contact topology, Proc. of the ICM 1998, Doc. Math. (1998), 327-338.

[E-G-H] Y. Eliashberg, A. Givental and H. Hofer, Introduction to symplectic field theory. Preprint

[E-G] Y. Eliashberg and M. Gromov, Convex symplectic manifolds. In Several complex variables and complex geometry, Part 2 (Santa Cruz, CA, 1989), 135-162, Proc. Sympos. Pure Math., 52, Part 2, Amer. Math. Soc., Providence, RI, 1991.

[E-K-L] L. Ein, O. Küchle and R. Lazarsfeld, Local positivity of ample line bundles. J. Diff. Geom. 42, (1995) 193-219.

[E-L] L. Ein and R. Lazarsfeld, Seshadri constants on smooth surfaces. Astérisque 218, 177186, (1993).

[Fu] W. Fulton, Intersection theory. Second edition. Springer-Verlag, 1998.

[Gri] P. Griffiths, Hermitian differential geometry, Chern classes, and positive vector bundles. In, Global Analysis (Papers in Honor of K. Kodaira), 185-251 Univ. Tokyo Press, Tokyo, 1969.

[G-H] P. Griffiths and J. Harris, Principles of algebraic geometry. Pure and Applied Mathematics. Wiley-Interscience (John Wiley and Sons), New York, 1978.

[Gr-1] M. Gromov, Partial Differential Relations. Springer-Verlag, (1986).

[Gr-2] M. Gromov, Pseudoholomorphic curves in symplectic manifolds. Invent. Math. 82 (1985), no. 2, 307-347.

$[\mathrm{H}-\mathrm{V}] \quad \mathrm{H}$. Hofer and C. Viterbo, The Weinstein conjecture in the presence of holomorphic spheres, Comm. Pure Appl. Math. 45 (1992), 583-622.

[H-Z] H. Hofer and E. Zehnder, Symplectic Invariants and Hamiltonian dynamics, Birkhäuser Verlag, Basel, 1994.

[La] F. Lalonde, Isotopy of symplectic balls, Gromov's radius and the structure of ruled symplectic 4-manifolds, Math. Ann. 300 (1994), no. 2, 273-296.

[Lau] F. Laudenbach, Homotopie régulière inactive et engouffrement symplectique, Ann. Inst. Fourier (Grenoble) 36 (1986), no. 2, 93-111.

[Laz] R. Lazarsfeld, Lengths of Periods and Seshadri Constants of Abelian Varieties, Math. Res. Let. 3 (1996), 439-47.

[McD-1] D. McDuff, Blow ups and symplectic embeddings in dimension 4, Topology 30 (1991), no. 3, 409-421.

[McD-2] D. McDuff, Remarks on the uniqueness of symplectic blowing up, in: Symplectic geometry, 157-167, London Math. Soc. Lecture Note Ser. 192, Cambridge Univ. Press, Cambridge, 1993.

[McD-3] D. McDuff, From symplectic deformation to isotopy, Topics in symplectic 4-manifolds (Irvine, CA, 1996), 85-99, First Int. Press Lect. Ser., I, Internat. Press, Cambridge, MA, 1998.

[M-P] D. McDuff and L. Polterovich, Symplectic packings and algebraic geometry, Invent. Math. 115 (1994), no. 3, 405-434.

[M-S] D. McDuff and D. Salamon, Introduction to symplectic topology, Second edition, Oxford Mathematical Monographs, Oxford University Press, New York, 1998.

[Oh-1] Y.-G. Oh, Floer cohomology of Lagrangian intersections and pseudo-holomorphic disks. I, Comm. Pure Appl. Math. 46 (1993), no. 7, 949-993.

[Oh-2] Y.-G. Oh, Floer cohomology, spectral sequences, and the Maslov class of Lagrangian embeddings, Internat. Math. Res. Notices 7 (1996), 305-346.

[Po] L. Polterovich, Monotone Lagrange submanifolds of linear spaces and the Maslov class in cotangent bundles, Math. Z. 207 (1991), no. 2, 217-222. 
[Se] P. Seidel, Graded Lagrangian submanifolds, Bull. Soc. Math. France 128 (2000), no. 1, 103-149.

[Vi-1] C. Viterbo, Functors and computations in Floer homology with applications. I, Geom. Funct. Anal. 9 (1999), no. 5, 985-1033.

[Vi-2] C. Viterbo, Properties of embedded Lagrange manifolds, In: First European Congress of Mathematics, Vol. II (Paris, 1992), 463-474, Progr. Math. 120, Birkhäuser, Basel, 1994.

[Vi-3] C. Viterbo, Symplectic real algebraic geometry, Preprint.

[We] A. Weinstein, Lectures on Symplectic manifolds. CBMS Conference Series, 29. American Mathematical Society, Providence, RI, 1977.

Paul Biran

School of Mathematical Sciences

Tel-Aviv University

Ramat-Aviv

Tel-Aviv 69978

Israel

e-mail: biran@math.tau.ac.il

(Received: November 13, 2000)
Kai Cieliebak

Stanford University

Department of Mathematics

Stanford CA 94305-2125

USA

e-mail: kai@math.stanford.edu

To access this journal online:

4D) http://www.birkhauser.ch 